\title{
Effects of Control on the AC-Side Admittance of a Modular Multilevel Converter
}

\author{
Luca Bessegato, Student Member, IEEE, Kalle Ilves, Member, IEEE, Lennart Harnefors, Fellow, IEEE, \\ Staffan Norrga, Member, IEEE,
}

\begin{abstract}
The stability of a modular multilevel converter connected to an ac grid can be assessed by analyzing the converter ac-side admittance in relation to the grid impedance. The converter control parameters have a strong impact on the admittance and they can be adjusted for achieving system stability. This paper focuses on the admittance-shaping effect produced by different current-control schemes, either designed on a per-phase basis or in the $d q$ frame using space vectors. A linear analytical model of the converter ac-side admittance is developed, including the different current-control schemes and the phase-locked loop. Different solutions for computing the insertion indices are also analyzed, showing that for a closed-loop scheme a compact expression of the admittance is obtained. The impact of the control parameters on the admittance is discussed and verified experimentally, giving guidelines for designing the system in terms of stability. Moreover, recommendations on whether a simplified admittance expression could be used instead of the detailed model are given. The findings from the admittanceshaping analysis are used to recreate a grid-converter system whose stability is determined by the control parameters. The developed admittance model is then used in this experimental case study, showing that the stability of the interconnected system can be assessed using the Nyquist stability criterion.
\end{abstract}

Index Terms-Modular multilevel converters, admittance, current control, stability, frequency-domain analysis, linearization techniques.

\section{INTRODUCTION}

$\mathbf{M}$ ODULAR multilevel converters (MMCs) [1], [2] are a class of topologies that has received notable attention in recent years, both from the scientific community and from industry. Modularity, scalability, low power losses, and low harmonic distortion are the main features that make MMCs suitable for high-power applications, such as voltage source converter (VSC) based HVDC systems, static synchronous compensators (STATCOMs), medium-voltage motor drives, and static traction converters [3]-[6]. The advantages offered by MMCs come at the expense of an inherently complex behavior, which has motivated research on several aspects of the MMCs, such as dynamic performance [7], [8], modulation techniques [9]-[11], modeling and control [4], [5], steady-state analysis [12]-[14], operation under unbalanced grid conditions [15], submodule implementations [16], and many others.

A topic on which research is still growing is the stability analysis of the interconnected system, formed by the MMC

Manuscript received August 1, 2018; revised October 22, 2018; accepted October 25, 2018.

L. Bessegato and S. Norrga are with the Royal Institute of Technology (KTH), Stockholm 100 44, Sweden (e-mail: lucabe@kth.se; norrga@kth.se).

K. Ilves and L. Harnefors are with ABB Corporate Research, Västerås 721 78, Sweden (e-mail: kalle.ilves@ se.abb.com; lennart.harnefors@se.abb.com). and the ac grid. For instance, an MMC designed for operating with an infinite bus may function incorrectly when connected to a weak ac grid, potentially causing instability. The stability of the interconnected system can be analyzed using the impedance-based stability criterion [17] or the passivity-based stability assessment [18], which are based on the expression of the converter impedance, or admittance, measured at its ac terminals. Modeling the MMC ac-side admittance is a challenging task, due to the converter dynamic behavior, which is highly nonlinear and generates several frequency components in the spectra of the converter variables. For this reason, the results documented for the two-level VSC topology [19], [20] cannot be directly extended to the MMC.

The state-of-the-art in MMC ac-side admittance modeling is summarized as follows. The authors of [21] and [22] derive an analytical model of the MMC ac-side admittance by using small-signal analysis in the time domain, which is then shifted to the Laplace domain where the admittance expression is obtained. Reference [23] presents an MMC impedance model based on harmonic linearization, considering three perturbation frequency components and solving the resulting equations analytically by elimination of variables; yet, the derivation is mathematically intricate and cannot be easily adjusted to include additional frequency components, different control schemes, or the phase-locked loop (PLL). Later in [24] and [25], the same authors derive an MMC impedance model based on harmonic state-space modeling; still, the ac-side current control and the PLL are not incorporated into the analysis. Reference [26] proposes a sequence impedance model for MMCs, based on multiharmonic linearization. Among the existing literature, this work is the most thorough and complete; however, it involves significant matrix manipulation as it is not designed for keeping the complexity to a minimum level.

Given the state-of-the-art in MMC ac-side admittance modeling, the authors of the present paper have developed in [27] a method for calculating the MMC ac-side admittance. In the study, a linear model is obtained by analyzing the main perturbation frequency components of the converter variables individually. The proposed method is aimed at achieving good accuracy while keeping the complexity to a minimum level; furthermore, it is designed for being easily adjustable for analyzing different control schemes and different frequency components.

Using the previously developed method as foundation, this paper aims to incorporate the current control into the analysis, assessing its impact on the MMC ac-side admittance. The shaping effects that the control parameters have on the ad- 
mittance are discussed, giving useful guidelines for designing the system in terms of stability. Compared with the previously published results, the novelty in this work and its original contributions are:

- the inclusion of different current-control schemes into the admittance model, such as per-phase ac-side current control, $d q$ frame ac-side current control, and circulating current control;

- the incorporation of two different methods for computing the insertion indices, i.e., an open-loop and a closed-loop scheme, into the admittance analysis;

- an evaluation, accompanied by experimental verification, on how the individual terms of the current controller shape the admittance.

Moreover, the admittance model is further developed, including:

- an improved frequency-domain model of the PLL, needed for assessing the impact of the $d q$ transformation, and its inverse, on the admittance;

- the simplification of the admittance calculation by including only five perturbation frequency components and by using an approximated steady-state solution;

- a discussion on whether a simpler two-level VSC admittance model could be used instead of a detailed MMC admittance model.

Finally, this paper presents the analysis of an experimental case study, where the developed admittance model is used for assessing the stability of the grid-converter system, using a control parameter to transition between stable and unstable conditions.

The paper is organized as follows. Section II presents the model of the converter and the analyzed control schemes. Section III describes how to derive the admittance model for the different control schemes. Section IV discusses how current control shapes the converter admittance and presents how the admittance model can be used to assess the stability of the grid-converter interconnected system. Finally, Section V summarizes this work and its conclusions.

\section{System Model}

The MMC investigated in this paper, depicted in Fig. 1, is configured for three-phase ac to dc conversion, or vice versa. The converter topology consists of three upper arms and three lower arms, each comprised of $N$ submodules, typically halfbridge or full-bridge submodules, and an arm inductance $L$; in addition, a resistance $R$ is included to account for the losses in the arm.

The MMC model adopted in this study, described in [8], is based on time averaging, which neglects the switching operations, and it assumes that the submodule capacitances are balanced within the arm. The resulting MMC model, described in the following, is compact and functional, however, its validity is limited to the frequency region below the equivalent switching frequency [10], due to the time averaging.

The converter is modeled on a per-phase basis, dropping the subscript denoting the phase when not needed.

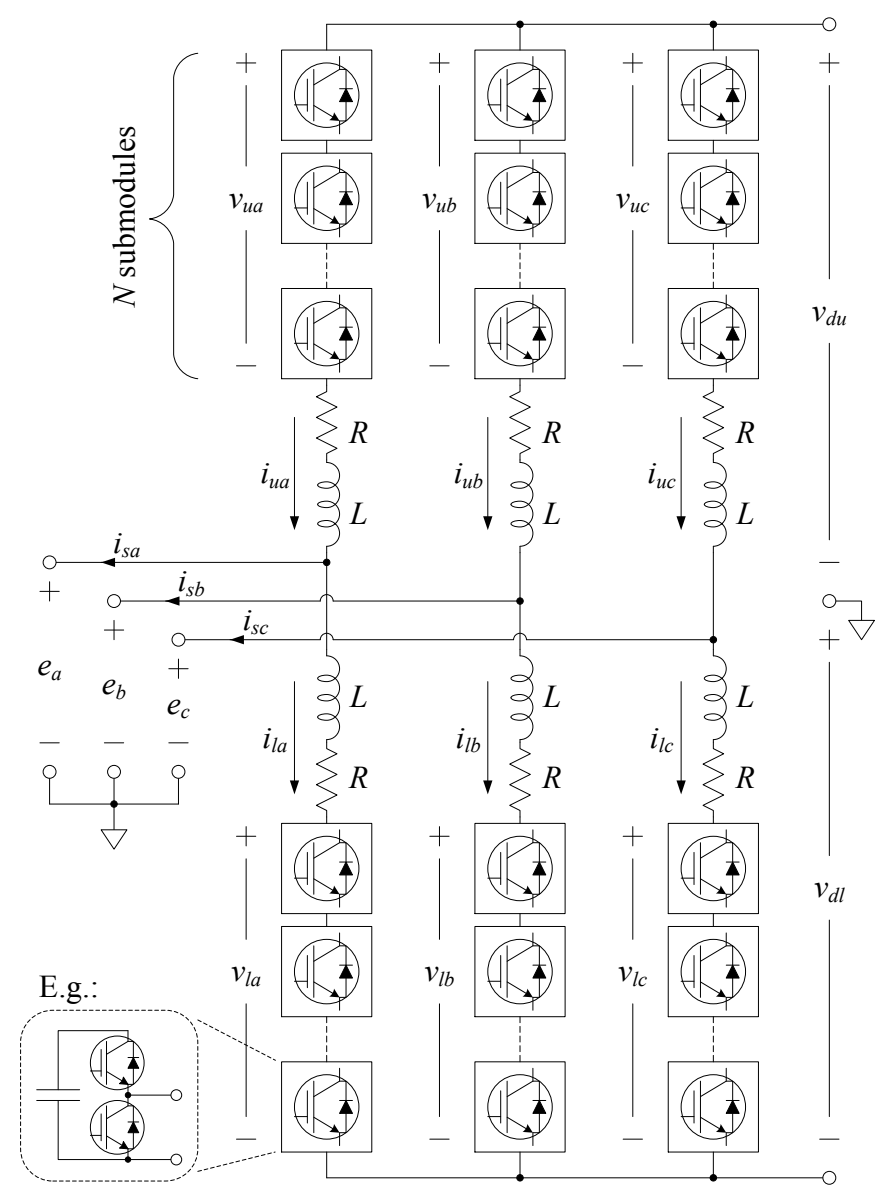

Fig. 1. Modular multilevel converter topology; a half-bridge submodule is shown as example of submodule implementation.

\section{A. Dynamics of the Converter}

The dynamic behavior of the converter is described using four variables: arm currents, arm voltages, sum capacitorvoltages, and insertion indices.

The arm-current dynamics are described using Kirchhoff's voltage law,

$$
\begin{gathered}
L \frac{d i_{u}}{d t}+R i_{u}=v_{d u}-v_{u}-e \\
L \frac{d i_{l}}{d t}+R i_{l}=v_{d l}-v_{l}+e,
\end{gathered}
$$

where $i_{u}$ is the upper-arm current, $i_{l}$ is the lower-arm current, $v_{u}$ is the upper-arm voltage, $v_{l}$ is the lower-arm voltage, $v_{d u}$ is the upper-dc-side voltage, $v_{d l}$ is the lower-dc-side voltage, and $e$ is the point-of-common-coupling (PCC) voltage

$$
e=e_{1} \cos [\vartheta(t)], \quad \text { with } \quad \vartheta(t)=\omega_{1} t=2 \pi f_{1} t .
$$

Using time averaging to neglect the switching operations, the arm voltages can be expressed as

$$
v_{u, l}=n_{u, l} v_{C u, l}^{\Sigma}
$$

where $n_{u, l}$ are the insertion indices and $v_{C u, l}^{\Sigma}$ are the sum capacitor-voltages. 
An expression of the sum capacitor-voltages can be obtained from the arm-capacitance energy and the instantaneous armpower, assuming balanced capacitor voltages [8], i.e.,

$$
v_{C u, l}^{\Sigma}=\frac{1}{C} \int n_{u, l} i_{u, l} d t+v_{C 0}^{\Sigma},
$$

where $v_{C 0}^{\Sigma}$ is the average sum-capacitor-voltage and $C$ is the arm capacitance, defined as the submodule capacitance divided by $N$.

The insertion indices are the signals used for controlling the converter; they can be computed as fixed references or using feedback control. A description of the control schemes analyzed in this paper is presented in the following subsection.

\section{B. Control of the Converter}

The employed control schemes are based on the following transformation of the arm currents [4] [8]

$$
i_{s}=i_{u}-i_{l} \quad i_{c}=\frac{i_{u}+i_{l}}{2},
$$

where $i_{s}$ is the ac-side current and $i_{c}$ is the circulating current. Using these definitions, and assuming $v_{d u}=v_{d l}=v_{d}$, (1)-(2) are rewritten as

$$
\begin{array}{ll}
\frac{L}{2} \frac{d i_{s}}{d t}+\frac{R}{2} i_{s}=v_{s}-e & \text { with } v_{s}=\frac{-v_{u}+v_{l}}{2} \\
L \frac{d i_{c}}{d t}+R i_{c}=\frac{v_{d}}{2}-v_{c} & \text { with } v_{c}=\frac{v_{u}+v_{l}}{2},
\end{array}
$$

where $v_{s}$ is the voltage driving $i_{s}$ and $v_{c}$ is the voltage driving $i_{c}$. This approach allows for controlling $i_{s}$ and $i_{c}$ independently, as two separate current controllers can be designed using (7)-(8).

The voltage references resulting from the current controllers, $v_{s}^{\star}$ and $v_{c}^{\star}$, are used for computing the insertion indices. Two solutions are studied: an open-loop and a closedloop scheme. In the first alternative, the voltage references are divided by the dc-bus voltage reference $v_{d}^{\star}$, i.e.,

$$
n_{u}=\frac{v_{c}^{\star}-v_{s}^{\star}}{v_{d}^{\star}} e^{-s T_{d}} \quad n_{l}=\frac{v_{c}^{\star}+v_{s}^{\star}}{v_{d}^{\star}} e^{-s T_{d}},
$$

where $T_{d}$ is the time delay of the control system. This scheme is simple and inherently gives an asymptotically stable system, meaning that an arm-balancing controller is not required [28]. Undesired harmonic components, which appear in the arm-voltages and the arm-currents as a drawback of this approach, can easily be suppressed using resonant controllers in the current controllers [29]. In the closed-loop scheme, the voltage references are divided by the measured sum-capacitorvoltages, i.e.,

$$
n_{u}=\frac{v_{c}^{\star}-v_{s}^{\star}}{v_{C u}^{\Sigma}} e^{-s T_{d}} \quad n_{l}=\frac{v_{c}^{\star}+v_{s}^{\star}}{v_{C l}^{\Sigma}} e^{-s T_{d}} .
$$

Substituting (10) into (4) shows that this scheme computes ideal insertion indices, as $v_{C u}^{\Sigma}$ and $v_{C l}^{\Sigma}$ cancel out and the arm voltages match their references (except for the control system time delay). However, with this scheme the sum-capacitor voltages are marginally stable and an arm-balancing controller is required [30].
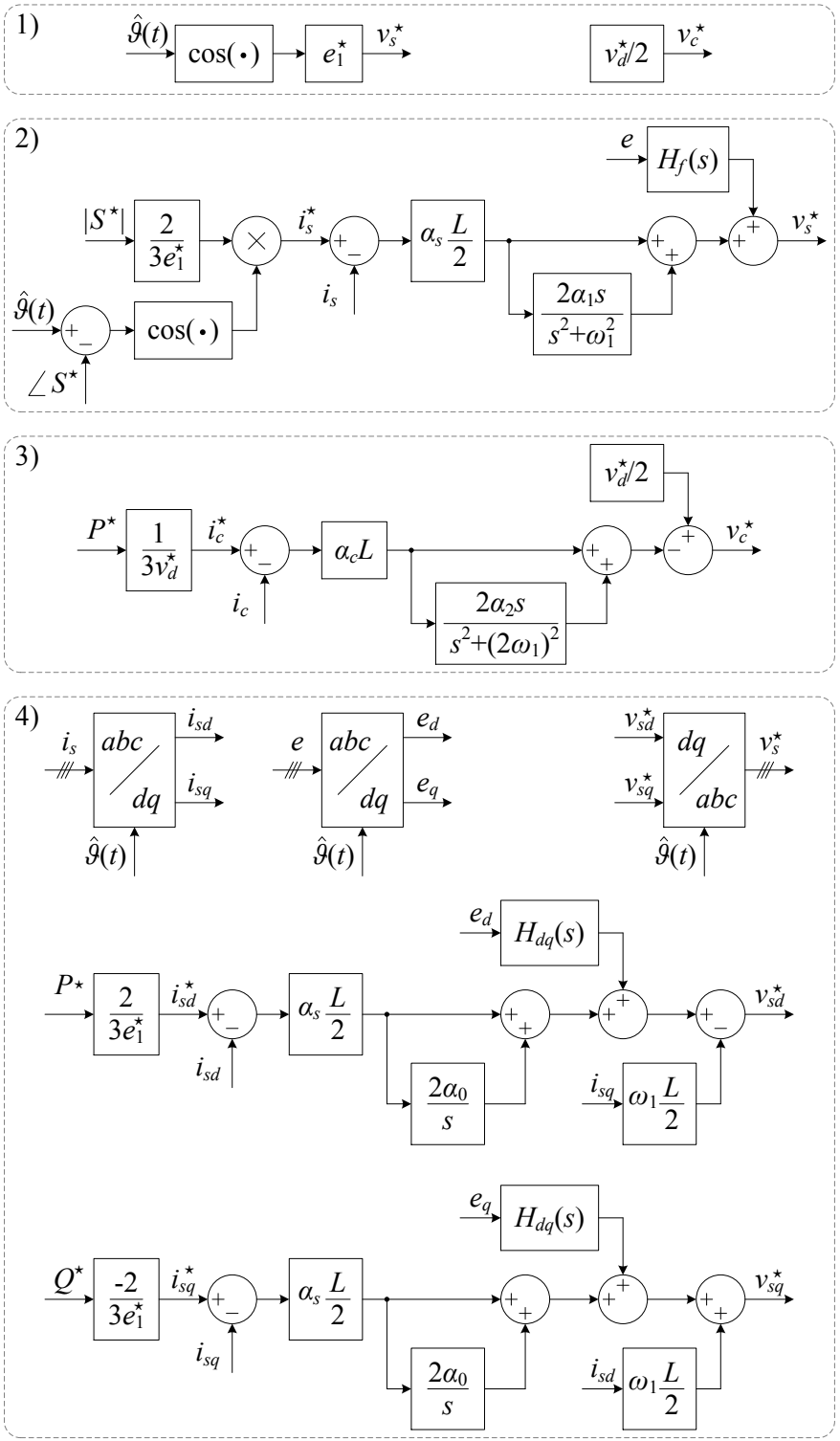

Fig. 2. Block diagrams of the different MMC control schemes: 1) fixed references; 2) per-phase ac-side current control; 3) circulating current control; and 4) $d q$ frame ac-side current control. For cases 1), 2), and 3) only phase $a$ is shown.

As the purpose of this study is to evaluate the effect of different current controllers on the MMC ac-side admittance, several control solutions are examined, which are described in the following and shown in Fig. 2

1) Fixed References: This is the most basic control scheme applicable to the MMC. The voltage references are generated in an open-loop fashion, with $v_{s}^{\star}$ and $v_{c}^{\star}$ setting the ac and dc components of the arm voltages, respectively, i.e.,

$$
v_{s}^{\star}=e_{1}^{\star} \cos [\hat{\vartheta}(t)] \quad v_{c}^{\star}=\frac{v_{d}^{\star}}{2},
$$

where $e_{1}^{\star}$ is the PCC-voltage amplitude reference and $\hat{\vartheta}(t)$ is the estimate of the PCC-voltage angle, provided by the PLL. Although this control scheme has limited applicability, it is a useful reference case for this study, as it shows the MMC ac-side admittance in absence of a closed-loop current control. 
2) Per-Phase AC-Side Current Control: In this configuration, an ac-side current controller is implemented for each phase in stationary coordinates [28]. It consists of: 1) a proportional controller, which sets the closed-loop-system bandwidth to $\left.\alpha_{s} ; 2\right)$ a resonant controller, which allows for tracking of the ac-side current reference $i_{s}^{\star}$; and 3) a feedforward of the PCC voltage, which improves the dynamic performance, i.e.,

$$
v_{s}^{\star}=F_{s}(s)\left(i_{s}^{\star}-i_{s}\right)+H_{f}(s) e,
$$

where $F_{s}(s)$ is the proportional-resonant controller

$$
F_{s}(s)=\alpha_{s} \frac{L}{2}\left(1+\frac{2 \alpha_{1} s}{s^{2}+\omega_{1}^{2}}\right)
$$

and $H_{f}(s)$ is a band-pass filter with bandwidth $\alpha_{f}$

$$
H_{f}(s)=\frac{\alpha_{f} s}{s^{2}+\alpha_{f} s+\omega_{1}^{2}} .
$$

The ac-side current reference $i_{s}^{\star}$ is set from the complex-power reference $S^{\star}$ as

$$
i_{s}^{\star}=\frac{2\left|S^{\star}\right|}{3 e_{1}^{\star}} \cos \left[\hat{\vartheta}(t)-\angle S^{\star}\right] \quad S^{\star}=P^{\star}+j Q^{\star},
$$

where $P^{\star}$ and $Q^{\star}$ are the active- and reactive-power references.

3) Circulating Current Control: The circulating current controller consists of: 1) a feedforward term $v_{d}^{\star} / 2$, which sets the dc component of the arm voltages; 2) a proportional controller, which sets the closed-loop-system bandwidth to $\alpha_{c}$; and 3) a resonant controller, which allows for suppressing the second-order harmonic in the circulating current, i.e.,

$$
v_{c}^{\star}=\frac{v_{d}^{\star}}{2}-F_{c}(s)\left(i_{c}^{\star}-i_{c}\right),
$$

where $F_{c}(s)$ is the proportional-resonant controller

$$
F_{c}(s)=\alpha_{c} L\left(1+\frac{2 \alpha_{2} s}{s^{2}+\left(2 \omega_{1}\right)^{2}}\right) .
$$

The circulating current reference $i_{c}^{\star}$ is set from the activepower reference, neglecting the losses in the converter, i.e.,

$$
i_{c}^{\star}=\frac{P^{\star}}{3 v_{d}^{\star}}
$$

4) DQ Frame AC-Side Current Control: In this configuration, the ac-side current space vector is used to design a current controller in synchronous coordinates, i.e., in the $d q$ frame. The measured ac-side current and PCC voltage are transformed into the $d q$ frame and used in the controller, which consists of: 1) a proportional controller, which sets the closedloop-system bandwidth to $\alpha_{s} ; 2$ ) an integral controller, which allows for tracking of the $d q$ components of the ac-side current reference, $i_{s d}^{\star}$ and $i_{s q}^{\star}$;3) a feedforward of the $d q$ components of the PCC voltage; and 4) a decoupling term, i.e.,

$$
\begin{aligned}
& v_{s d}^{\star}=F_{d q}(s)\left(i_{s d}^{\star}-i_{s d}\right)+H_{d q}(s) e_{d}-\omega_{1} \frac{L}{2} i_{s q} \\
& v_{s q}^{\star}=F_{d q}(s)\left(i_{s q}^{\star}-i_{s q}\right)+H_{d q}(s) e_{q}+\omega_{1} \frac{L}{2} i_{s d},
\end{aligned}
$$

where $F_{d q}(s)$ is the proportional-integral controller

$$
F_{d q}(s)=\alpha_{s} \frac{L}{2}\left(1+\frac{2 \alpha_{1}}{s}\right)
$$

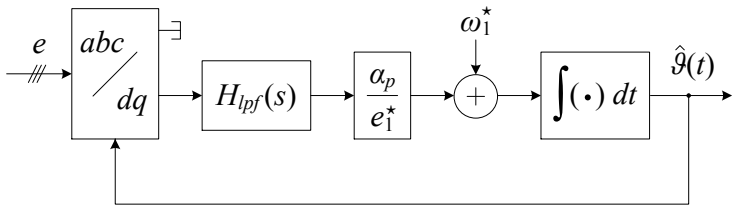

Fig. 3. Block diagram of the PLL.

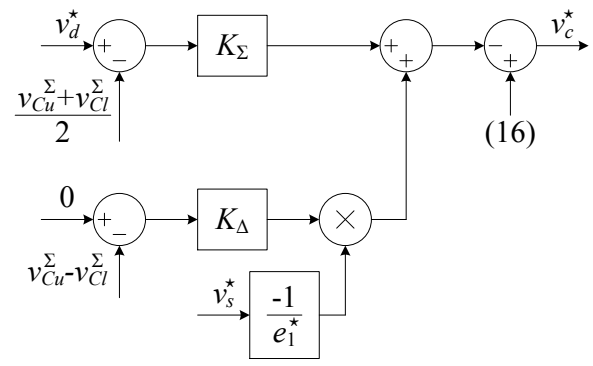

Fig. 4. Block diagram of the arm-balancing controller.

and $H_{d q}(s)$ is a low-pass filter with bandwidth $\alpha_{f}$

$$
H_{d q}(s)=\frac{\alpha_{f}}{s+\alpha_{f}} .
$$

The $d q$ components of the ac-side current reference are set from the active- and reactive-power references as

$$
i_{s d}^{\star}=\frac{2 P^{\star}}{3 e_{1}^{\star}} \quad i_{s q}^{\star}=-\frac{2 Q^{\star}}{3 e_{1}^{\star}} .
$$

After (19)-(20), the voltage references $v_{s d}^{\star}$ and $v_{s q}^{\star}$ are reverted into the stationary coordinates, obtaining $v_{s}^{\star}$ for each phase, which are used in (9) for calculating the insertion indices.

\section{PLL}

The PLL provides the controller with the estimate $\hat{\vartheta}(t)$ of the PCC voltage angle $\vartheta(t)$, which is used in every control scheme, either for generating the sinusoidal references (11) and (15) or for performing the $d q$ transformation and its inverse.

The PLL, shown in Fig. 3, consists of a feedback loop built around an $a b c / d q$ transformation, which drives the $q$ signal to zero as $\hat{\vartheta}(t)$ tracks $\vartheta(t)$. The PLL feedback loop includes: 1) a low pass filter $\left.H_{l p f}(s) ; 2\right)$ a proportional controller, which sets the closed-loop-system bandwidth to $\alpha_{p} ; 3$ ) a feedforward of the fundamental angular frequency reference $\omega_{1}^{\star}$; and 4) an integrator.

\section{Arm-Balancing Controller}

When the insertion indices are computed using a closedloop scheme, asymptotic stability of the sum-capacitor voltages is ensured by the arm-balancing controller [30], shown in Fig. 4. The controller consists of two parts, which control the average and the imbalance sum-capacitor-voltages, defined as

$$
v_{C}^{\Sigma}=\frac{v_{C u}^{\Sigma}+v_{C l}^{\Sigma}}{2} \quad v_{C}^{\Delta}=v_{C u}^{\Sigma}-v_{C l}^{\Sigma},
$$



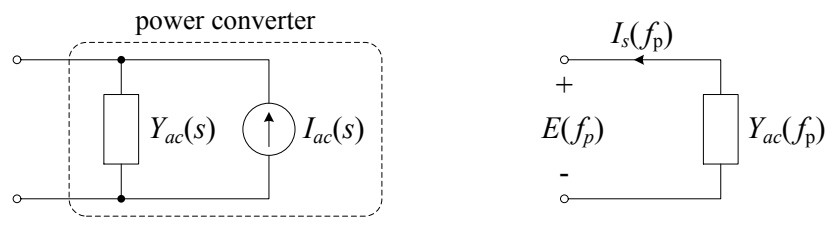

Fig. 5. Small-signal representation of the power converter using its Norton equivalent circuit (left); equivalent circuit at perturbation frequency used for calculating the ac-side admittance of the converter (right).

to $v_{d}^{\star}$ and 0 respectively. The first part of the controller drives a $\mathrm{dc}$ term in the circulating current, which multiplies the dc component of $n_{u}$ and $n_{l}$ in (5), thus controlling the average sumcapacitor-voltage. Similarly, the second part of the controller drives a fundamental-frequency term in the circulating current, which is in phase with $n_{u}$ and in antiphase with $n_{l}$. That term multiplies the fundamental-frequency component of $n_{u}$ and $n_{l}$ in (5), resulting in a de term that controls the imbalance sum-capacitor-voltage. The arm-balancing controller modifies the voltage reference (16) as

$v_{c}^{\star}=\frac{v_{d}^{\star}}{2}-F_{c}(s)\left(i_{c}^{\star}-i_{c}\right)-K_{\Sigma}\left(v_{d}^{\star}-v_{C}^{\Sigma}\right)+K_{\Delta} v_{C}^{\Delta}\left(-\frac{v_{s}^{\star}}{e_{1}^{\star}}\right)$,

where $K_{\Sigma}$ and $K_{\Delta}$ are proportional gains and $-v_{s}^{\star} / e_{1}^{\star}$ produces a fundamental-frequency component in phase with $n_{u}$.

\section{AC-Side Admittance Calculation}

When using the impedance-based stability criterion [17], the ac-side of the power converter is modeled by its Norton equivalent circuit, consisting of a current source in parallel with an admittance, as shown in Fig. 5. In this linear representation, valid for small-signal analysis, the current source $I_{a c}(s)$ embodies the steady-state value of the ac-side current, whereas the admittance $Y_{a c}(s)$ models the frequency response of the converter.

In order to calculate the admittance, a small-signal perturbation is superimposed on the PCC voltage

$$
e=e_{1} \cos \left(\omega_{1} t\right)+e_{p} \cos \left(\omega_{p} t\right) \quad E\left(f_{p}\right) \ll E\left(f_{1}\right),
$$

where $E\left(f_{1}\right)=e_{1} / 2$ denotes the Fourier coefficient of $E$ at $f_{1}$. Using the equivalent circuit at perturbation frequency, shown in Fig. 5, the admittance can be calculated as

$$
Y_{a c}\left(f_{p}\right)=-\frac{I_{s}\left(f_{p}\right)}{E\left(f_{p}\right)},
$$

i.e., as ratio between the current response to the applied voltage perturbation.

Calculating the current response $I_{s}\left(f_{p}\right)$ is not straightforward, due to the nonlinear behavior of the MMC, caused by the multiplications in (4) and (5). Applying the perturbation $E\left(f_{p}\right)$ affects the frequency spectra of the converter variables, generating new frequency components. For example, multiplying a variable at $f_{p}$ with a variable at $f_{1}$ results in the two
TABLE I

\begin{tabular}{|c|c|c|}
\hline Symbol & Variable & Obtainable from \\
\hline$i_{u, l}$ & arm currents & Kirchhoff's voltage law \\
\hline$v_{u, l}$ & arm voltages & $\begin{array}{l}\text { time averaging of the switching op- } \\
\text { erations }\end{array}$ \\
\hline$v_{C u, l}^{\Sigma}$ & sum capacitor-voltages & $\begin{array}{l}\text { arm-capacitance energy and instan- } \\
\text { taneous arm-power, assuming bal- } \\
\text { anced capacitor voltages }\end{array}$ \\
\hline$n_{u, l}$ & insertion indices & output of the control algorithm \\
\hline
\end{tabular}

CONVERTER VARIABLES

new components $f_{p}-f_{1}$ and $f_{p}+f_{1}$, i.e.,

$$
\begin{aligned}
x \cdot y= & {\left[x_{p} \cos \left(\omega_{p} t+\varphi_{p}\right)\right]\left[y_{1} \cos \left(\omega_{1} t+\varphi_{1}\right)\right] } \\
= & \frac{1}{2} x_{p} y_{1}\left\{\cos \left[\left(\omega_{p}-\omega_{1}\right) t+\varphi_{p}-\varphi_{1}\right]\right. \\
& \left.+\cos \left[\left(\omega_{p}+\omega_{1}\right) t+\varphi_{p}+\varphi_{1}\right]\right\} .
\end{aligned}
$$

Because of this, the current response $I_{s}\left(f_{p}\right)$ cannot be calculated using the classic linearization method, where all the frequency components except for $f_{p}$ are discarded, as it would lead to a highly inaccurate result. Instead, an extension of the classic linearization method, called harmonic linearization [31], is used for tackling the problem. Here, a linear model is built not only using the components at $f_{p}$, but including a whole set of frequency components. Specifically, combinations of $f_{p}$ with steady-state components are taken into account, whereas harmonics of $f_{p}$ are neglected, due to the assumption that $E\left(f_{p}\right)$ is small. In the following subsection, a description of the linear model built to obtain the MMC ac-side admittance is presented. Further details about the adopted method can be found in [27].

\section{A. Frequency-Domain Analysis of the MMC Variables}

The converter variables, summarized in Table I, are analyzed in the frequency domain to obtain a linear analytical model of the MMC ac-side admittance. The frequency components included in the analysis are chosen as the minimum amount necessary to achieve the desired accuracy in the results, in order to keep the complexity to a minimum level. Two steadystate values and five perturbation values are chosen, i.e.,

$$
f=\left(0, f_{1}, f_{p}, f_{p} \pm f_{1}, f_{p} \pm 2 f_{1}\right) .
$$

The analysis can be further simplified by exploiting the inherent symmetries of the MMC topology. Hence, only the upper arm of phase $a$ is analyzed.

The expressions at $f_{p}$ are presented in this section, while the expressions at $f=\left(f_{p} \pm f_{1}, f_{p} \pm 2 f_{1}\right)$ are given in the Appendix, as their derivations are similar. The steady-state components of the converter variables are also given in the Appendix.

1) Upper-Arm Current: The Kirchhoff's voltage law (1) is expressed at $f_{p}$, giving

$$
I_{u}\left(f_{p}\right)=-\frac{V_{u}\left(f_{p}\right)}{j \omega_{p} L+R}-\frac{E\left(f_{p}\right)}{j \omega_{p} L+R} .
$$


TABLE II

FREQUENCY COMPONENTS RESUlTING FROM A MULTIPLICATION: THE PERTURbation Frequency Components (First COLumn) COMbine With THE STEAdY-STATE COMPONENTS (FIRST ROW) THROUGH ADDITION AND SUBTRACTION (EXPRESSED USING $-f_{1}$ )

\begin{tabular}{c|c|c|c}
\hline \hline & $-f_{1}$ & 0 & $f_{1}$ \\
\hline$f_{p}-2 f_{1}$ & $f_{p}-3 f_{1}$ & $f_{p}-2 f_{1}$ & $f_{p}-f_{1}$ \\
$f_{p}-f_{1}$ & $f_{p}-2 f_{1}$ & $f_{p}-f_{1}$ & $f_{p}$ \\
$f_{p}$ & $f_{p}-f_{1}$ & $f_{p}$ & $f_{p}+f_{1}$ \\
$f_{p}+f_{1}$ & $f_{p}$ & $f_{p}+f_{1}$ & $f_{p}+2 f_{1}$ \\
$f_{p}+2 f_{1}$ & $f_{p}+f_{1}$ & $f_{p}+2 f_{1}$ & $f_{p}+3 f_{1}$ \\
\hline \hline
\end{tabular}

2) Upper-Arm Voltage: In the frequency domain, the multiplication in (4) causes the frequency components of $n_{u}$ and $v_{C u}^{\Sigma}$ to combine through addition and subtraction. Since the analysis is restricted to the frequency components listed in (29), Table II is used to identify the combinations that result in the frequencies of interest. For instance, $f_{p}$ is obtained from $\left(f_{p}-f_{1}\right)+f_{1}, f_{p}+0$, and $\left(f_{1}+f_{p}\right)-f_{1}$. When using the negative counterpart of a frequency component (e.g., $\left.-f_{1}\right)$, the complex conjugate of the Fourier coefficient is used, due to the Hermitian symmetry property of the frequency components of real-valued functions, i.e., $X(-f)=\overline{X(f)}$. Hence, expressing (4) at $f_{p}$ results in

$$
\begin{aligned}
& V_{u}\left(f_{p}\right)=V_{C u}^{\Sigma}\left(f_{p}\right) N_{u}(0)+N_{u}\left(f_{p}\right) V_{C u}^{\Sigma}(0) \\
& \quad+V_{C u}^{\Sigma}\left(f_{p}-f_{1}\right) N_{u}\left(f_{1}\right)+N_{u}\left(f_{p}-f_{1}\right) V_{C u}^{\Sigma}\left(f_{1}\right) \\
& \quad+V_{C u}^{\Sigma}\left(f_{p}+f_{1}\right) \overline{N_{u}\left(f_{1}\right)}+N_{u}\left(f_{p}+f_{1}\right) \overline{V_{C u}^{\Sigma}\left(f_{1}\right)} .
\end{aligned}
$$

The steady-state values of $n_{u}$ and $v_{C u}^{\Sigma}$ are calculated beforehand (see the Appendix); therefore, they appear in (31) as coefficients, meaning that (31) is linear.

3) Upper-Arm Sum-Capacitor-Voltage: Equation (5) contains a multiplication between $n_{u}$ and $i_{u}$, which causes the two frequency spectra to combine. The reasoning used to obtain (31) can also be applied here, leading to

$$
\begin{aligned}
& V_{C u}^{\Sigma}\left(f_{p}\right)=\frac{1}{j \omega_{p} C}\left[I_{u}\left(f_{p}\right) N_{u}(0)+N_{u}\left(f_{p}\right) I_{u}(0)\right. \\
& \quad+I_{u}\left(f_{p}-f_{1}\right) N_{u}\left(f_{1}\right)+N_{u}\left(f_{p}-f_{1}\right) I_{u}\left(f_{1}\right) \\
& \left.\quad+I_{u}\left(f_{p}+f_{1}\right) \overline{N_{u}\left(f_{1}\right)}+N_{u}\left(f_{p}+f_{1}\right) \overline{I_{u}\left(f_{1}\right)}\right] .
\end{aligned}
$$

4) Upper-Arm Insertion Index: For the open-loop scheme, equation (9) is expressed for $f=\left(f_{p}, f_{p} \pm f_{1}, f_{p} \pm 2 f_{1}\right)$ as

$$
N_{u}(f)=\frac{V_{c}^{\star}(f)-V_{s}^{\star}(f)}{v_{d}^{\star}} e^{-j 2 \pi f T_{d}},
$$

where $V_{s}^{\star}(f)$ and $V_{c}^{\star}(f)$ depend on the chosen control scheme. A frequency-domain analysis of the voltage references $v_{s}^{\star}$ and $v_{c}^{\star}$ for the different control schemes is presented in the following. The effects of the closed-loop scheme (10) on the ac-side admittance are analyzed in Section III-D.

\section{B. Frequency-Domain Analysis of the Control Variables}

Before examining the different control schemes individually, a linear frequency-domain model of the PLL is needed. The PLL has a noticeable impact on the MMC ac-side admittance,

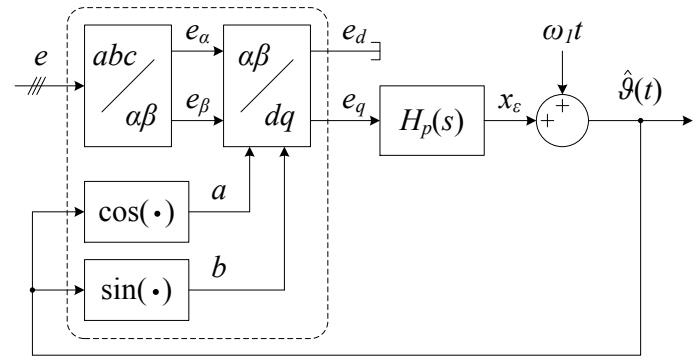

Fig. 6. Block diagram used for the PLL frequency-domain analysis.

especially when using $d q$ frame current control, therefore it must be modeled with care.

Fig. 6 shows the block diagram used for the PLL frequencydomain analysis. The main variables and frequency components of interest are:

- the PCC voltage $e$, whose frequency components are $E\left(f_{1}\right)$ and $E\left(f_{p}\right)$

- the variable $x_{\varepsilon}$, modeling the perturbation at the output of the PLL, expressed in the frequency domain as $X_{\varepsilon}\left(f_{p}-f_{1}\right)$, where the frequency shift by $-f_{1}$ is due to the $a b c / d q$ transformation;

- the variables $a=\cos [\hat{\vartheta}(t)]$ and $b=\sin [\hat{\vartheta}(t)]$, used in the transformation.

Firstly, the effects of the $a b c / d q$ transformation in the frequency domain are analyzed. The variables $a$ and $b$ are linearized for $x_{\varepsilon} \simeq 0$ as

$$
\begin{aligned}
a & =\cos \left(\omega_{1} t+x_{\varepsilon}\right)=\cos \left(\omega_{1} t\right) \cos \left(x_{\varepsilon}\right)-\sin \left(\omega_{1} t\right) \sin \left(x_{\varepsilon}\right) \\
& \simeq \cos \left(\omega_{1} t\right)-\sin \left(\omega_{1} t\right) x_{\varepsilon} \\
b & =\sin \left(\omega_{1} t+x_{\varepsilon}\right)=\sin \left(\omega_{1} t\right) \cos \left(x_{\varepsilon}\right)+\cos \left(\omega_{1} t\right) \sin \left(x_{\varepsilon}\right) \\
& \simeq \sin \left(\omega_{1} t\right)+\cos \left(\omega_{1} t\right) x_{\varepsilon} .
\end{aligned}
$$

The linearized $a$ and $b$ can be expressed using complex exponentials as

$$
\begin{aligned}
& a=\frac{e^{j \omega_{1} t}+e^{-j \omega_{1} t}}{2}-\frac{e^{j \omega_{1} t}-e^{-j \omega_{1} t}}{2 j} x_{\varepsilon} \\
& b=\frac{e^{j \omega_{1} t}-e^{-j \omega_{1} t}}{2 j}+\frac{e^{j \omega_{1} t}+e^{-j \omega_{1} t}}{2} x_{\varepsilon} .
\end{aligned}
$$

In these expressions, $x_{\varepsilon}$ is multiplied by the complex exponentials $e^{j \omega_{1} t}$ and $e^{-j \omega_{1} t}$; in the frequency domain, this corresponds to a frequency shift by $f_{1}$ and $-f_{1}$, respectively. Using the previous expressions, the frequency components of $a$ and $b$ result

$$
\begin{array}{ll}
A\left(f_{1}\right)=\frac{1}{2} & B\left(f_{1}\right)=\frac{1}{2 j} \\
A\left(f_{p}\right)=-\frac{X_{\varepsilon}\left(f_{p}-f_{1}\right)}{2 j} & B\left(f_{p}\right)=\frac{X_{\varepsilon}\left(f_{p}-f_{1}\right)}{2} \\
A\left(f_{p}-2 f_{1}\right)=\frac{X_{\varepsilon}\left(f_{p}-f_{1}\right)}{2 j} & B\left(f_{p}-2 f_{1}\right)=\frac{X_{\varepsilon}\left(f_{p}-f_{1}\right)}{2} .
\end{array}
$$

The PCC voltage is transformed in the $d q$ frame as

$$
e_{d}=e_{\alpha} a+e_{\beta} b \quad e_{q}=-e_{\alpha} b+e_{\beta} a,
$$


leading to the perturbation frequency components

$$
\begin{aligned}
E_{d}\left(f_{p}-f_{1}\right)= & E_{\alpha}\left(f_{p}\right) \overline{A\left(f_{1}\right)}+E_{\beta}\left(f_{p}\right) \overline{B\left(f_{1}\right)} \\
& +A\left(f_{p}\right) \overline{E_{\alpha}\left(f_{1}\right)}+B\left(f_{p}\right) \overline{E_{\beta}\left(f_{1}\right)} \\
& +A\left(f_{p}-2 f_{1}\right) E_{\alpha}\left(f_{1}\right)+B\left(f_{p}-2 f_{1}\right) E_{\beta}\left(f_{1}\right)
\end{aligned}
$$

$$
\begin{aligned}
E_{q}\left(f_{p}-f_{1}\right)= & -E_{\alpha}\left(f_{p}\right) \overline{B\left(f_{1}\right)}+E_{\beta}\left(f_{p}\right) \overline{A\left(f_{1}\right)} \\
& -B\left(f_{p}\right) \overline{E_{\alpha}\left(f_{1}\right)}+A\left(f_{p}\right) \overline{E_{\beta}\left(f_{1}\right)} \\
& -B\left(f_{p}-2 f_{1}\right) E_{\alpha}\left(f_{1}\right)+A\left(f_{p}-2 f_{1}\right) E_{\beta}\left(f_{1}\right) .
\end{aligned}
$$

With $E_{\alpha}(f)=E(f)$ and $E_{\beta}(f)=-j E(f)$, for $f=\left(f_{1}, f_{p}\right)$, and using (38)-(40), the previous expressions are simplified as

$$
\begin{aligned}
& E_{d}\left(f_{p}-f_{1}\right)=E\left(f_{p}\right)+2 \operatorname{Im}\left[E\left(f_{1}\right)\right] X_{\varepsilon}\left(f_{p}-f_{1}\right) \\
& E_{q}\left(f_{p}-f_{1}\right)=-j E\left(f_{p}\right)-2 \operatorname{Re}\left[E\left(f_{1}\right)\right] X_{\varepsilon}\left(f_{p}-f_{1}\right) .
\end{aligned}
$$

The signals $e_{q}$ and $x_{\varepsilon}$ are related through the PLL open-loop transfer function $H_{p}(s)$, defined as

$$
H_{p}(s)=\frac{X_{\varepsilon}(s)}{E_{q}(s)}=\frac{\alpha_{p}}{e_{1}^{\star}} \frac{H_{l p f}(s)}{s} .
$$

Finally, using (45) with $E_{q}\left(f_{p}-f_{1}\right)$ expressed using (46), the perturbation frequency component $X_{\varepsilon}\left(f_{p}-f_{1}\right)$ at the output of the PLL results

$$
X_{\varepsilon}\left(f_{p}-f_{1}\right)=\frac{-j H_{p}\left[j\left(\omega_{p}-\omega_{1}\right)\right]}{1+2 \operatorname{Re}\left[E\left(f_{1}\right)\right] H_{p}\left[j\left(\omega_{p}-\omega_{1}\right)\right]} E\left(f_{p}\right) .
$$

Using the Fourier coefficients $E\left(f_{1}\right)=e_{1} / 2$ and $E\left(f_{p}\right)=$ $e_{p} / 2$, the frequency components (47) and (38)-(40) are obtained. These components are then used in the following analysis to include the effects of the PLL on the control variables.

1) Fixed References: Equation (11) expresses $v_{s}^{\star}$ as a function of $a=\cos [\hat{\vartheta}(t)]$, which contains perturbation frequency components at $f_{p}$ and $f_{p}-2 f_{1}$, i.e.,

$$
V_{s}^{\star}(f)=e_{1}^{\star} A(f) \quad \text { for } f=\left(f_{p}, f_{p}-2 f_{1}\right) .
$$

2) Per-Phase AC-Side Current Control: Due to the symmetries of the MMC topology, only components at $f_{p}$ and $f_{p} \pm 2 f_{1}$ appear in the ac-side current, with $I_{s}(f)=2 I_{u}(f)=-2 I_{l}(f)$, for $f=\left(f_{p}, f_{p} \pm 2 f_{1}\right)$. Equation (12) is then evaluated at these frequencies, resulting in

$$
\begin{aligned}
& V_{s}^{\star}\left(f_{p}\right)=F_{s}\left(j \omega_{p}\right)\left[I_{s}^{\star}\left(f_{p}\right)-I_{s}\left(f_{p}\right)\right]+H_{f}\left(j \omega_{p}\right) E\left(f_{p}\right) \\
& V_{s}^{\star}\left(f_{p}-2 f_{1}\right)=F_{s}\left[j\left(\omega_{p}-2 \omega_{1}\right)\right]\left[I_{s}^{\star}\left(f_{p}-2 f_{1}\right)-I_{s}\left(f_{p}-2 f_{1}\right)\right] \\
& V_{s}^{\star}\left(f_{p}+2 f_{1}\right)=-F_{s}\left[j\left(\omega_{p}+2 \omega_{1}\right)\right] I_{s}\left(f_{p}+2 f_{1}\right),
\end{aligned}
$$

with

$$
I_{s}^{\star}(f)=\frac{2\left(P^{\star}-j Q^{\star}\right)}{3 e_{1}^{\star}} A(f) \quad \text { for } f=\left(f_{p}, f_{p}-2 f_{1}\right) .
$$

3) Circulating Current Control: Due to the symmetries of the MMC topology, only components at $f_{p} \pm f_{1}$ appear in the circulating current, with $I_{c}\left(f_{p} \pm f_{1}\right)=I_{u}\left(f_{p} \pm f_{1}\right)=I_{l}\left(f_{p} \pm\right.$ $\left.f_{1}\right)$. Equation (16) is then evaluated at these frequencies, resulting in

$$
V_{c}^{\star}\left(f_{p} \pm f_{1}\right)=F_{c}\left[j\left(\omega_{p} \pm \omega_{1}\right)\right] I_{c}\left(f_{p} \pm f_{1}\right) .
$$

4) DQ Frame AC-Side Current Control: In the $d q$ frame, the only perturbation frequency component is at $f_{p}-f_{1}$. The $d q$ components of $i_{s}$ are obtained similarly to (44)-(45) as

$$
\begin{aligned}
I_{s d}\left(f_{p}-f_{1}\right)= & I_{s}\left(f_{p}\right)+I_{s}\left(f_{p}-2 f_{1}\right) \\
& +2 \operatorname{Im}\left[I_{s}\left(f_{1}\right)\right] X_{\varepsilon}\left(f_{p}-f_{1}\right) \\
I_{s q}\left(f_{p}-f_{1}\right)= & -j I_{s}\left(f_{p}\right)+j I_{s}\left(f_{p}-2 f_{1}\right) \\
& -2 \operatorname{Re}\left[I_{s}\left(f_{1}\right)\right] X_{\varepsilon}\left(f_{p}-f_{1}\right) .
\end{aligned}
$$

Equations (19)-(20) are expressed at $f_{p}-f_{1}$ as

$$
\begin{aligned}
& V_{s d}^{\star}\left(f_{p}-f_{1}\right)=-F_{d q}\left[j\left(\omega_{p}-\omega_{1}\right)\right] I_{s d}\left(f_{p}-f_{1}\right) \\
& \quad+H_{d q}\left[j\left(\omega_{p}-\omega_{1}\right)\right] E_{d}\left(f_{p}-f_{1}\right)-\frac{\omega_{1} L}{2} I_{s q}\left(f_{p}-f_{1}\right) \\
& V_{s q}^{\star}\left(f_{p}-f_{1}\right)=-F_{d q}\left[j\left(\omega_{p}-\omega_{1}\right)\right] I_{s q}\left(f_{p}-f_{1}\right) \\
& \quad+H_{d q}\left[j\left(\omega_{p}-\omega_{1}\right)\right] E_{q}\left(f_{p}-f_{1}\right)+\frac{\omega_{1} L}{2} I_{s d}\left(f_{p}-f_{1}\right) .
\end{aligned}
$$

Then, the voltage references $v_{s d}^{\star}$ and $v_{s q}^{\star}$ are reverted into the stationary coordinates as

$$
v_{s \alpha}^{\star}=v_{s d}^{\star} a-v_{s q}^{\star} b \quad v_{s \beta}^{\star}=v_{s d}^{\star} b+v_{s q}^{\star} a,
$$

resulting into the frequency components

$$
\begin{aligned}
V_{s}^{\star}\left(f_{p}\right)= & \frac{1}{2} V_{s d}^{\star}\left(f_{p}-f_{1}\right)+\frac{j}{2} V_{s q}^{\star}\left(f_{p}-f_{1}\right) \\
& +A\left(f_{p}\right)\left[V_{s d}^{\star}(0)+j V_{s q}^{\star}(0)\right] \\
V_{s}^{\star}\left(f_{p}-\right. & \left.2 f_{1}\right)=\frac{1}{2} V_{s d}^{\star}\left(f_{p}-f_{1}\right)-\frac{j}{2} V_{s q}^{\star}\left(f_{p}-f_{1}\right) \\
& +A\left(f_{p}-2 f_{1}\right)\left[V_{s d}^{\star}(0)-j V_{s q}^{\star}(0)\right] .
\end{aligned}
$$

\section{Solution}

The expressions describing the frequency components of the converter variables at $f=\left(f_{p}, f_{p} \pm f_{1}, f_{p} \pm 2 f_{1}\right)$, i.e., (30)-(33), (72)-(83), and (54)-(57), are used to build a linear system with 24 equations and 24 unknown variables of the form

$$
A_{p} \mathbf{x}_{p}=B_{p},
$$

where the matrix $A_{p}$ contains the coefficients of the linear system, the vector $B_{p}$ contains the constant terms, and the vector $\mathbf{x}_{p}$ contains the system variables

$$
\begin{aligned}
\mathbf{x}_{p}=[ & I_{u}\left(f_{p}\right), I_{u}\left(f_{p}-f_{1}\right), I_{u}\left(f_{p}+f_{1}\right), I_{u}\left(f_{p}-2 f_{1}\right), \\
& I_{u}\left(f_{p}+2 f_{1}\right), V_{u}\left(f_{p}\right), V_{u}\left(f_{p}-f_{1}\right), V_{u}\left(f_{p}+f_{1}\right), \\
& V_{u}\left(f_{p}-2 f_{1}\right), V_{u}\left(f_{p}+2 f_{1}\right), V_{C u}^{\Sigma}\left(f_{p}\right), V_{C u}^{\Sigma}\left(f_{p}-f_{1}\right), \\
& V_{C u}^{\Sigma}\left(f_{p}+f_{1}\right), V_{C u}^{\Sigma}\left(f_{p}-2 f_{1}\right), V_{C u}^{\Sigma}\left(f_{p}+2 f_{1}\right), \\
& N_{u}\left(f_{p}\right), N_{u}\left(f_{p}-f_{1}\right), N_{u}\left(f_{p}+f_{1}\right), N_{u}\left(f_{p}-2 f_{1}\right), \\
& N_{u}\left(f_{p}+2 f_{1}\right), I_{s d}\left(f_{p}-f_{1}\right), I_{s q}\left(f_{p}-f_{1}\right), \\
& \left.V_{s d}^{\star}\left(f_{p}-f_{1}\right), V_{s q}^{\star}\left(f_{p}-f_{1}\right)\right]^{T} .
\end{aligned}
$$

The steady-state values of the converter variables are calculated beforehand (see Appendix); therefore, they appear as coefficients into $A_{p}$ and $B_{p}$.

The linear system (61) is solved to obtain $\mathbf{x}_{p}$. Due to the symmetries of the MMC topology $I_{s}\left(f_{p}\right)=2 I_{u}\left(f_{p}\right)$; 
therefore, the newly-found $I_{u}\left(f_{p}\right)$ is used to obtain the MMC ac-side admittance using (27) as

$$
Y_{a c}\left(f_{p}\right)=-\frac{2 I_{u}\left(f_{p}\right)}{E\left(f_{p}\right)} .
$$

\section{Admittance When Using the Closed-Loop Scheme}

As discussed in Section II-B, when computing the insertion indices with the closed-loop scheme $v_{C u}^{\Sigma}$ and $v_{C l}^{\Sigma}$ in (4) and (10) cancel out, leading to

$$
v_{s}=v_{s}^{\star} e^{-s T_{d}} \quad v_{c}=v_{c}^{\star} e^{-s T_{d}} .
$$

This greatly simplifies the admittance calculation, as (64) links the current-controller dynamics with the current dynamics (7) and the sum-capacitor voltages no longer appear in the calculation. Effectively, the MMC ac-side admittance now coincides with the ac-side admittance of a two-level VSC [20], with a VSC phase inductance of $L / 2$.

An analytical expression of the ac-side admittance of the MMC operated with the closed-loop scheme is obtained by substituting (59) into (7) using (64), also evaluated at $f_{p}$, and by using (54)-(57). After some algebraic manipulation, the following closed-form expression is obtained

$$
Y_{a c}\left(f_{p}\right)=\frac{1+\left(H_{P L L}-H_{d q}\left[j\left(\omega_{p}-\omega_{1}\right)\right]\right) e^{-j \omega_{p} T_{d}}}{\frac{j \omega_{p} L+R}{2}+\left(F_{d q}\left[j\left(\omega_{p}-\omega_{1}\right)\right]-\frac{j \omega_{1} L}{2}\right) e^{-j \omega_{p} T_{d}}},
$$

where $H_{P L L}$ groups the effects of the PLL on the admittance, introduced by the $d q$ transformation and its inverse, i.e.,

$$
\begin{aligned}
H_{P L L}= & {\left[\left(-F_{d q}\left[j\left(\omega_{p}-\omega_{1}\right)\right]+\frac{j \omega_{1} L}{2}\right) I_{s}\left(f_{1}\right)\right.} \\
& \left.+H_{d q}\left[j\left(\omega_{p}-\omega_{1}\right)\right] E\left(f_{1}\right)-V_{s}^{\star}\left(f_{1}\right)\right] \frac{j X_{\varepsilon}\left(f_{p}-f_{1}\right)}{E\left(f_{p}\right)} .
\end{aligned}
$$

A noteworthy implication of (64) is that neither the circulating current nor the circulating current controller influence the ac-side admittance, meaning that the closed-loop scheme effectively decouples the ac and the dc sides of the converter.

\section{VERIFICATION AND DISCUSSION}

The analytical admittance model presented in Section III is validated through experiments, showing how different control schemes shape the MMC ac-side admittance. Then, a case study showing how to assess the stability of a grid-converter system using the admittance model is presented.

\section{A. Admittance Shaping Using Current Control}

The experimental setup used to validate the admittance model is shown in Fig. 7 and Fig. 8. A down-scaled MMC prototype is connected at the PCC to a two-level inverter, which generates a small-signal perturbation superimposed on the fundamental-frequency three-phase voltage, as defined in (26). The perturbation frequency is programmable, therefore several measurements are made to obtain the frequencydomain plot of the admittance. At every iteration, $e$ and $i_{s}$

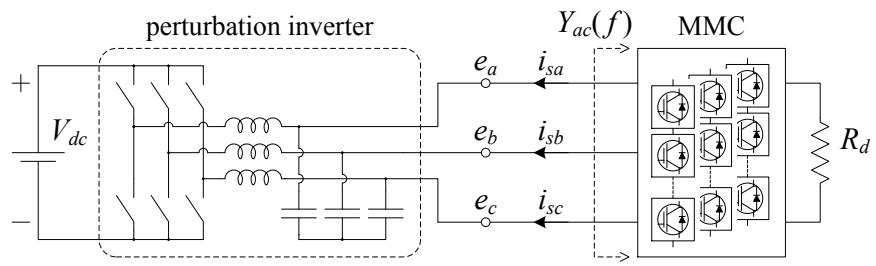

Fig. 7. Configuration of the experimental setup for measuring the MMC ac-side admittance.

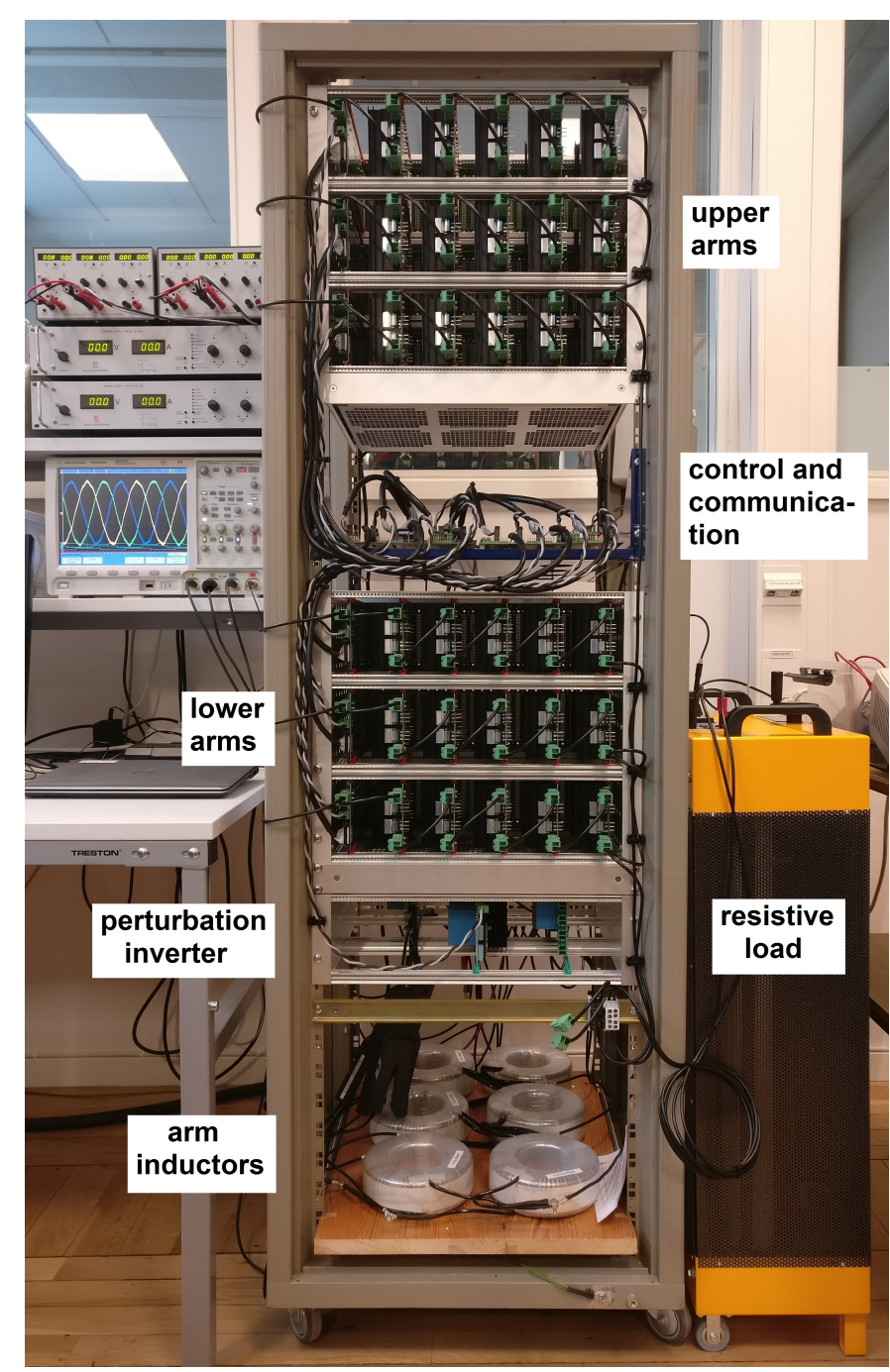

Fig. 8. Photograph of the experimental setup.

are measured to extract their component at $f_{p}$, to obtain the corresponding admittance value using (27).

The down-scaled MMC prototype, described in detail in [32], has five full-bridge submodules per arm, for a total of 30 submodules, and has a rated power of $10 \mathrm{~kW}$. Its control system is based on Xilinx Zynq-7000 system-on-chip, which integrates a programmable logic with a processing system. The chosen modulation scheme is the phase-shifted carrier PWM, which ensures the balancing of the individual capacitor voltages and symmetrical operating conditions between the arms, given that the carrier frequency is a noninteger multiple of $f_{1}$ [33]. The parameters of the experimental setup and the 
TABLE III

PARAMETERS OF THE EXPERIMENTAL SETUP

\begin{tabular}{l|l|l}
\hline \hline PCC voltage amplitude & $e_{1}$ & $48 \mathrm{~V}$ \\
Fundamental frequency & $f_{1}$ & $50 \mathrm{~Hz}$ \\
Resistive load & $R_{d}$ & $25 \Omega$ \\
Arm inductance & $L$ & $5.7 \mathrm{mH}$ \\
Arm resistance & $R$ & $0.55 \Omega$ \\
Arm capacitance & $C$ & $0.54 \mathrm{mF}$ \\
Submodules per arm & $N$ & 5 \\
Perturbation amplitude & $e_{p}$ & $0.8 \mathrm{~V}$ \\
Perturbation frequency & $f_{p}$ & $2 \mathrm{~Hz}-1 \mathrm{kHz}$ \\
\hline \hline
\end{tabular}

TABLE IV

SETTINGS OF THE MMC CONTROLLER

\begin{tabular}{l|l|l}
\hline \hline Carrier frequency & $f_{c}$ & $763 \mathrm{~Hz}$ \\
Control system delay time & $T_{d}$ & $65.5 \mu \mathrm{s}$ \\
DC-bus voltage reference & $v_{d}^{\star}$ & $107 \mathrm{~V}$ \\
Active-power reference & $P^{\star}$ & $-455 \mathrm{~W}$ \\
Reactive-power reference & $Q^{\star}$ & $0 \mathrm{VAr}$ \\
PLL closed-loop-system bandwidth & $\alpha_{p}$ & $50 \mathrm{rad} / \mathrm{s}$ \\
PLL low-pass filter bandwidth & $\alpha_{l p f}$ & $250 \mathrm{rad} / \mathrm{s}$ \\
AC-side current closed-loop-system bandwidth & $\alpha_{s}$ & $1200 \mathrm{rad} / \mathrm{s}$ \\
AC-side current resonant/integral controller gain & $\alpha_{1}$ & $100 \mathrm{rad} / \mathrm{s}$ \\
PCC voltage feedforward filter bandwidth & $\alpha_{f}$ & $1000 \mathrm{rad} / \mathrm{s}$ \\
Circulating current closed-loop-system bandwidth & $\alpha_{c}$ & $500 \mathrm{rad} / \mathrm{s}$ \\
Circulating current resonant controller gain & $\alpha_{2}$ & $100 \mathrm{rad} / \mathrm{s}$ \\
Arm-balancing controller average-part gain & $K_{\Sigma}$ & 2 \\
Arm-balancing controller imbalance-part gain & $K_{\Delta}$ & 1 \\
\hline \hline
\end{tabular}

settings of the MMC controller are given in Table III and Table IV respectively. The results presented in this subsection are obtained computing the insertion indices using the open-loop scheme; the effects of the closed-loop scheme are analyzed in the next subsection.

The Bode diagrams of the MMC ac-side admittance for different control schemes, obtained from the measurements and from the analytical model, are shown in Fig. 9. From these results, it is observed that:

- in all cases, the analytical model (lines) is in agreement with the experiments (dots), validating the proposed method for MMC ac-side admittance calculation, presented in Section III-C;

- in comparison to the fixed references case (blue), using current control (yellow and red) radically reshapes the admittance, lowering the magnitude and increasing the phase rotation in the analyzed frequency range.

The observed admittance-shaping phenomena are the result of a series of contributions, caused by the single elements forming the current controllers (e.g., $\alpha_{s}, \alpha_{c}$, etc.). The impact of these elements is assessed as follows. Starting from the converter operated with fixed references (blue), the different control elements are added progressively, evaluating their individual impact at every stage, until the converter is operated with per-phase current control and circulating current control (yellow). From the resulting Bode diagrams, shown in Fig. 10
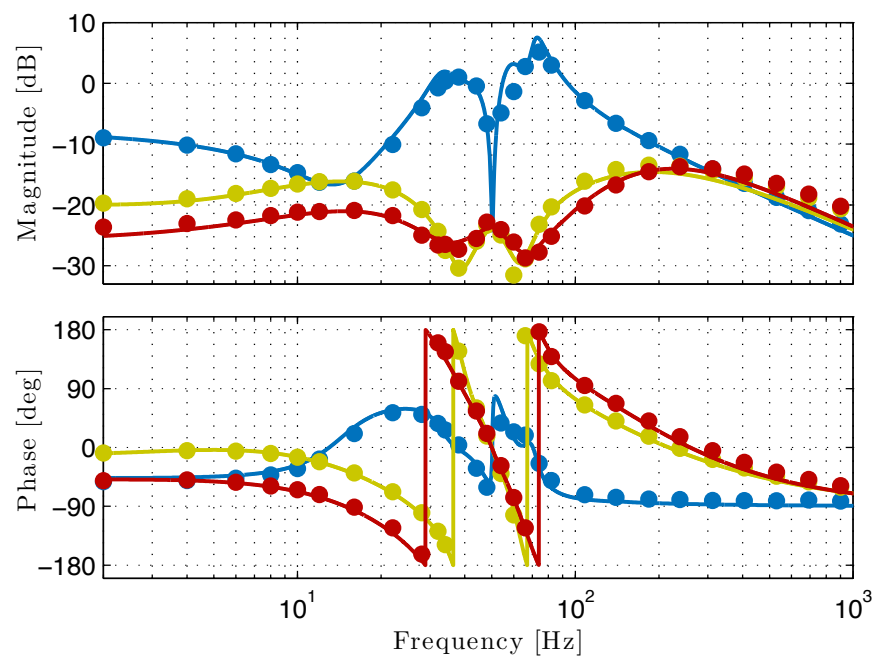

Fig. 9. Bode diagrams of the MMC ac-side admittance for different control schemes: fixed references (blue); per-phase ac-side current control and circulating current control (yellow); $d q$ frame ac-side current control and circulating current control (red). Results from the measurements (dots) and the analytical model (lines) are shown.
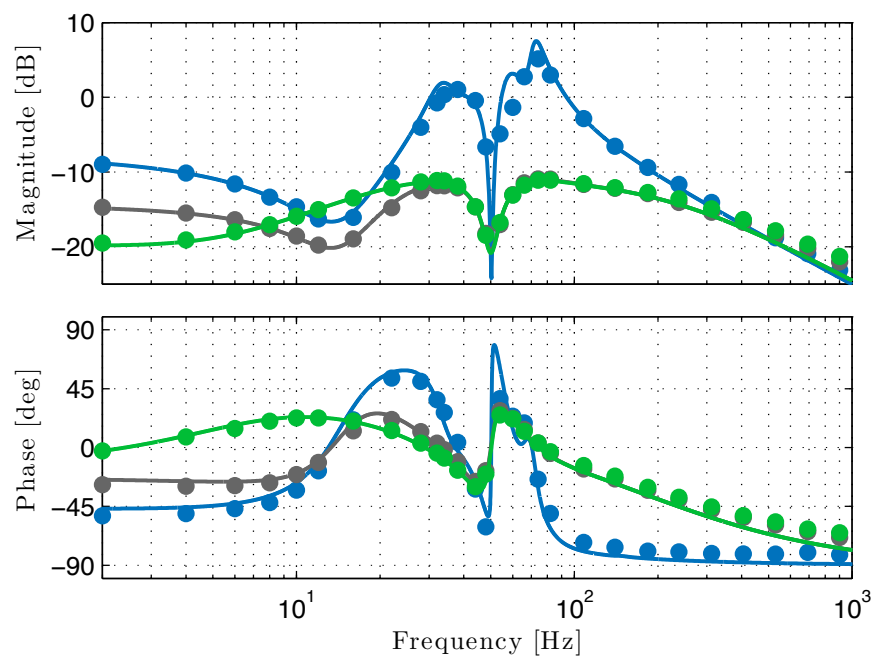

Fig. 10. Bode diagrams of the MMC ac-side admittance for different control schemes: fixed references (blue); addition of ac-side current proportional controller (grey); addition of circulating current controller (green). Results from the measurements (dots) and the analytical model (lines) are shown.

and Fig. 11, it is observed that:

- adding the ac-side current proportional controller (grey) greatly reduces the admittance magnitude, especially around fundamental frequency, and slightly modifies the phase angle diagram;

- adding the circulating current controller (green) slightly modifies the magnitude and the phase below fundamental frequency;

- adding the ac-side current resonant controller (purple) affects the admittance around fundamental frequency, accentuating the notch in the magnitude and the rotation in the phase;

- removing the fixed ac-voltage reference while adding the PCC voltage feedforward (yellow) widens the notch and 

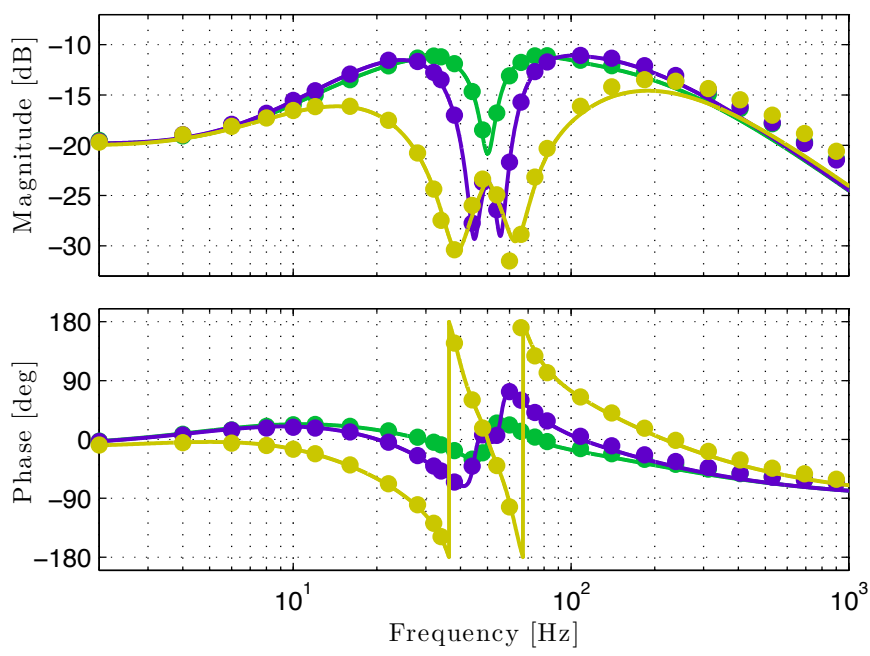

Fig. 11. Bode diagrams of the MMC ac-side admittance for different control schemes: fixed references with of ac-side current proportional controller and circulating current controller (green); addition of ac-side current resonant controller (purple); addition of the PCC voltage feedforward and removal of the fixed ac-voltage reference (yellow). Results from the measurements (dots) and the analytical model (lines) are shown.

greatly increases the phase rotation around fundamental frequency.

Typically, low magnitude and limited phase rotation are desired features in the converter admittance, as they improve the stability margin of the grid-converter interconnected system. Therefore, from the previous observations it is concluded that:

- the ac-side current proportional controller, thus, the closed-loop-system bandwidth $\alpha_{s}$, are highly beneficial, as they lower the admittance magnitude without degrading the phase;

- the circulating current controller has a limited impact on the admittance;

- the ac-side current resonant controller and the PCC voltage feedforward are double-edged blades, as they lower the admittance magnitude but also increase the phase rotation around fundamental frequency, meaning that they must be designed with care.

Analogous conclusions can be drawn for the converter operated with $d q$ frame ac-side current control (red curves in Fig. 9).

\section{B. Effect of the Closed-Loop Scheme}

The Bode diagrams of the MMC ac-side admittance for different computations of insertion indices, i.e., the open-loop and the closed-loop scheme, obtained from the measurements and from the analytical model, are shown in Fig. 12. From these results, it is observed that:

- also for the closed-loop scheme case, the analytical model (black line) is in agreement with the experiments (black dots), validating the derivation presented in Section III-D;

- above fundamental frequency the two cases overlap, meaning that the closed-form expression (65) can also be used for the open-loop scheme case, serving as a useful simplification;
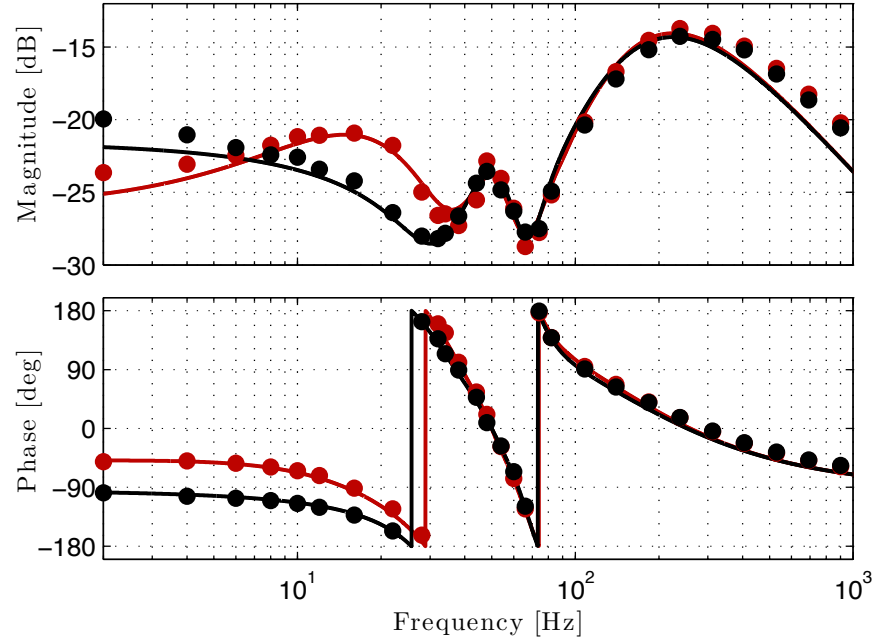

Fig. 12. Bode diagrams of the MMC ac-side admittance for different computations of insertion indices: open-loop scheme (red) and closed-loop scheme (black). In both cases the converter is operated with $d q$ frame ac-side current control and circulating current control; in the closed-loop scheme case the arm-balancing controller is used. Results from the measurements (dots) and the analytical model (lines) are shown.

- below fundamental frequency there is a noticeable difference between the two cases, both in the admittance magnitude and phase; therefore, when using the openloop scheme, it is recommended to obtain the admittance using the detailed model presented in Section III-C;

- in both cases the converter is operated with $d q$ frame acside current control and circulating current control; for the converter operated without current control (blue curves in Fig. 9) the detailed model should always be adopted.

\section{Stability Assessment Using the Admittance Model}

The admittance model presented in this study can be used for assessing the stability of a grid-converter system using the impedance-based stability criterion [17]. The Thevenin equivalent circuit of the ac grid, $V_{g}$ and $Z_{g}$, and the Norton equivalent circuit of the power converter, $I_{a c}$ and $Y_{a c}$, are used to form the circuit shown in Fig. 13, where the converter acside current is

$$
I_{s}(s)=\left[I_{a c}(s)-V_{g}(s) Y_{a c}(s)\right] \frac{1}{1+Z_{g}(s) Y_{a c}(s)} .
$$

Assuming that $I_{a c}, V_{g}$, and $Y_{a c}$ are stable, the stability of the interconnected system depends on the stability of the second term on the right-hand side of (67), which resembles the closed-loop expression of a negative feedback system. By linear control theory, this feedback system is stable if and only if $Z_{g}(s) Y_{a c}(s)$ satisfies the Nyquist stability criterion, i.e., the Nyquist plot of $Z_{g}(s) Y_{a c}(s)$ does not encircle the point $(-1+j 0)$ in the complex plane.

The impedance-based stability criterion is used in an experimental case study to verify its validity in presence of non-ideal characteristics, which are not included in the analytical model (e.g., parasitic components, switching operation, measurement noise, etc.). The experiment is designed as follows. A twolevel inverter and three inductors are used to emulate an ac 


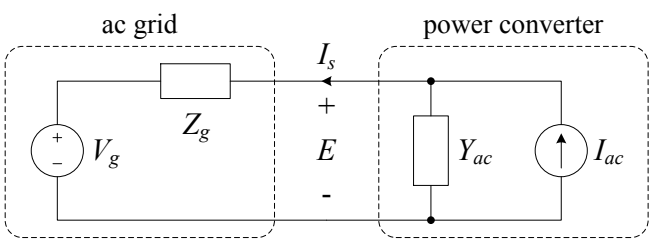

Fig. 13. Small-signal representation of the grid-converter interconnected system.

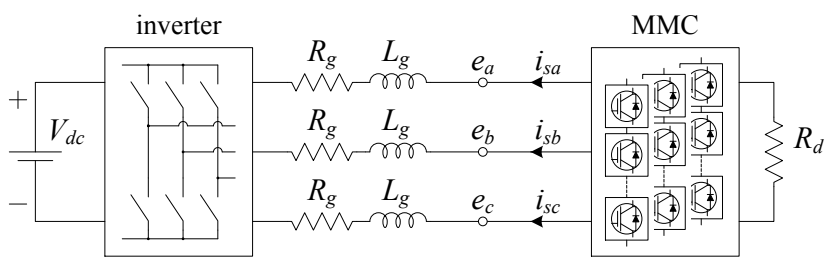

Fig. 14. Configuration of the experimental setup for assessing the stability of the grid-converter interconnected system.

grid with inductive impedance, $Z_{g}(s)=s L_{g}+R_{g}$, which is connected at the PCC to the down-scaled MMC prototype, as shown in Fig. 14. The MMC is operated with $d q$ frame ac-side current control and circulating current control, which exhibits an accentuated phase rotation around fundamental frequency, and the ac-side current closed-loop-system bandwidth $\alpha_{s}$ is used to adjust the admittance magnitude, thereby affecting the system stability. The ac-grid inductance and resistance are $L_{g}=10.2 \mathrm{mH}(0.42 \mathrm{pu})$ and $R_{g}=0.19 \Omega(0.025 \mathrm{pu})$; the other experiment parameters are the same, given in Table III and Table IV, and the insertion indices are computed using the open-loop scheme.

The system stability can be predicted using the Nyquist plot of $Z_{g}(s) Y_{a c}(s)$, presented in Fig. 15, which shows that:

- for $\alpha_{s}=1200 \mathrm{rad} / \mathrm{s}$ the point $(-1+j 0)$ is not encircled, meaning that the system is stable;

- for $\alpha_{s}=600 \mathrm{rad} / \mathrm{s}$ there is one clockwise encirclement of the point $(-1+j 0)$, meaning that the system is unstable.

The experimental setup is then operated with the two values of $\alpha_{s}$, in order to validate the theoretical analysis. Fig. 16 displays the measured $d q$ frame PCC voltage and ac-side current, which show that:

- for $\alpha_{s}=1200 \mathrm{rad} / \mathrm{s}$ the $d q$ frame waveforms are nearly constant, meaning that the system is stable;

- for $\alpha_{s}=600 \mathrm{rad} / \mathrm{s}$ the $d q$ frame waveforms oscillate, meaning that the system is unstable. The diverging trend, visible at $t=2 \mathrm{~s}$, is constrained by the insertion indices, which saturate at 0 and 1 , limiting the amplitude of the oscillations.

In addition, the waveforms of the converter variables during the experiment are shown in Fig. 17, for $\alpha_{s}=1200 \mathrm{rad} / \mathrm{s}$, and in Fig. 18, for $\alpha_{s}=600 \mathrm{rad} / \mathrm{s}$. In the unstable case, the measured oscillation frequency is $97 \mathrm{~Hz}$ in stationary coordinates and $47 \mathrm{~Hz}$ in synchronous coordinates.

Given the agreement between the theoretical analysis and the measured waveforms, it is concluded that the stability of the interconnected system can be predicted using the

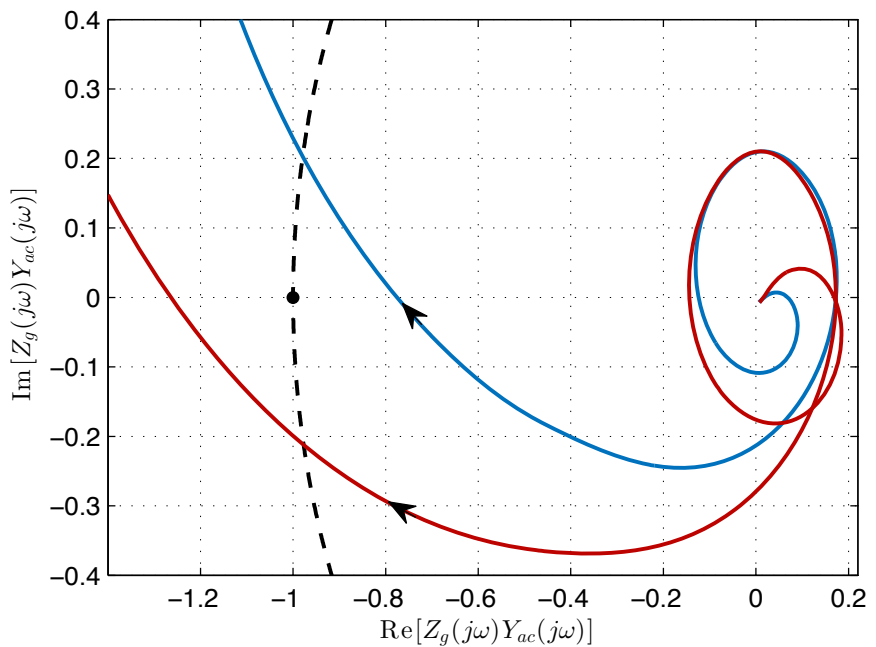

Fig. 15. Nyquist plot of $Z_{g}(s) Y_{a c}(s)$ for $\alpha_{s}=1200 \mathrm{rad} / \mathrm{s}$ (blue) and for $\alpha_{s}=600 \mathrm{rad} / \mathrm{s}$ (red). The unit circle and the point $(-1+j 0)$ are drawn in black.
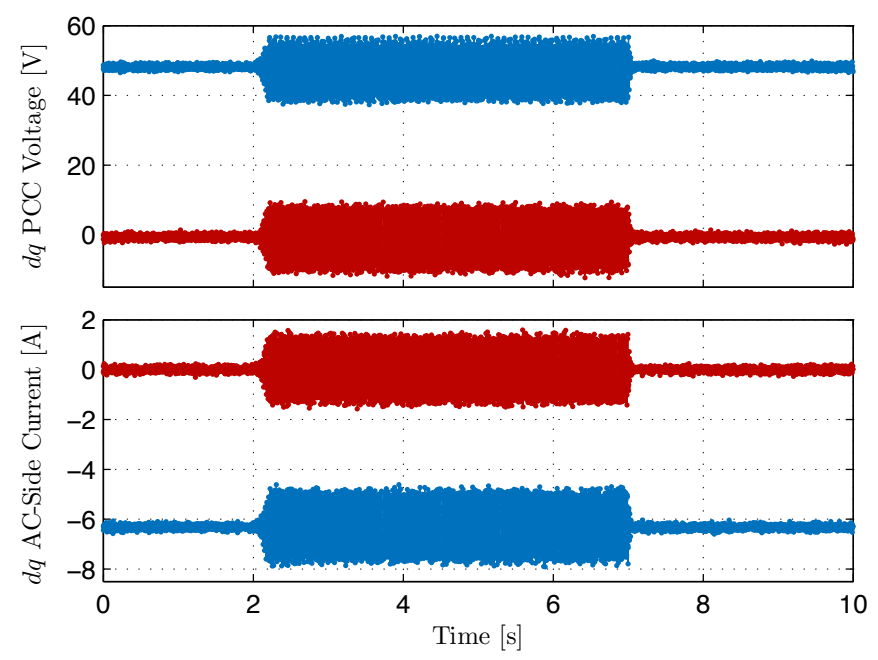

Fig. 16. Measured $d q$ frame PCC voltage (top) and ac-side current (bottom) during the stability assessment test. $\alpha_{s}$ is lowered from $1200 \mathrm{rad} / \mathrm{s}$ to $600 \mathrm{rad} / \mathrm{s}$ after $2 \mathrm{~s}$ and restored to $1200 \mathrm{rad} / \mathrm{s}$ after $7 \mathrm{~s}$. The $d$ components (blue) and the $q$ components (red) are saved from the MMC control system.

developed admittance model and the impedance-based stability criterion.

\section{CONClusion}

In this paper, different current-control schemes and different solution for computing the insertion indices are included in the linear analytical model of the MMC ac-side admittance. The frequency-domain model of the current-control schemes includes the effects of the PLL, which are significant especially when using $d q$ frame current control.

The admittance-shaping phenomena caused by using current control are discussed, validating the analytical study through experiments. In comparison to the converter operated with fixed references, generated in an open-loop fashion, using current control radically reshapes the admittance Bode diagram, lowering the magnitude and increasing the phase rotation in 

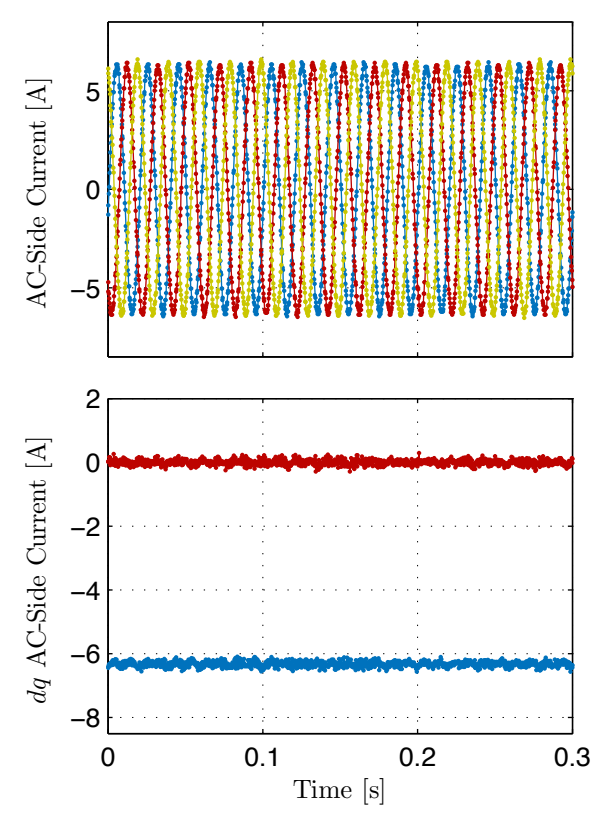
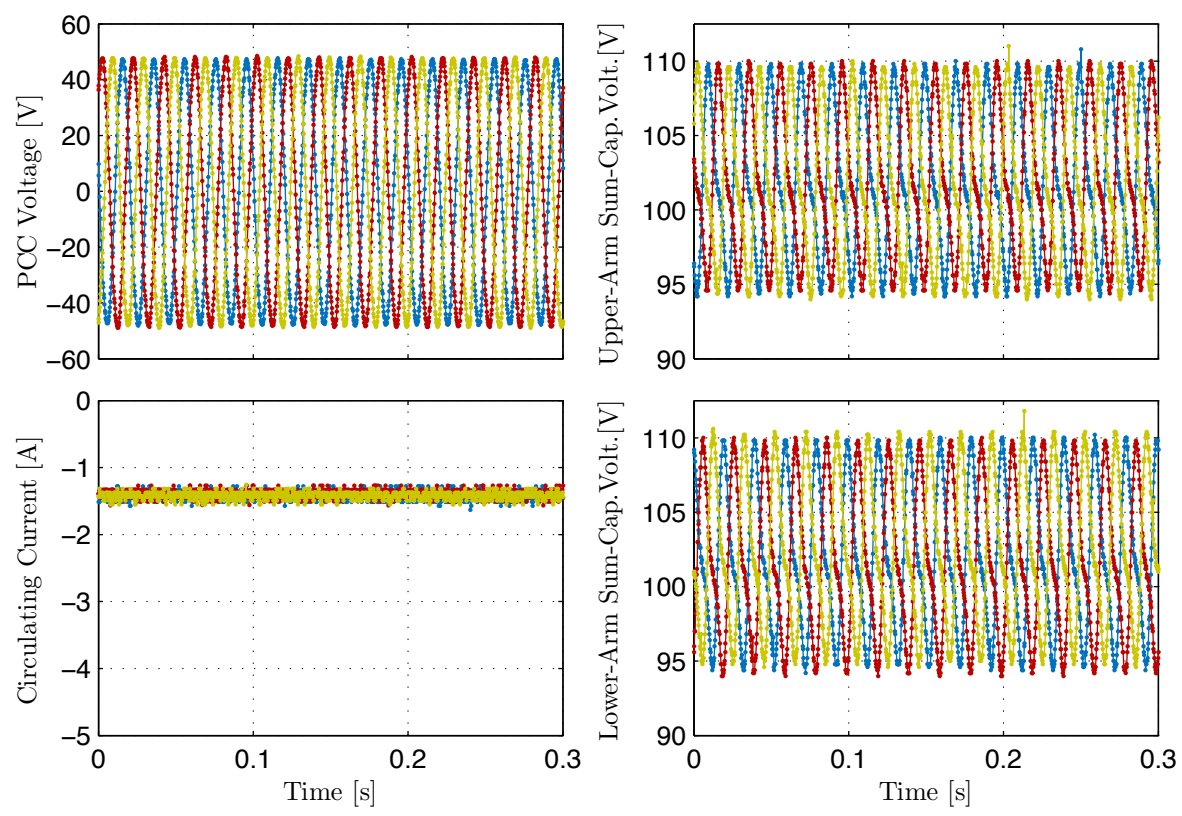

Fig. 17. Waveforms of the converter variables during the experiment with $\alpha_{s}=1200 \mathrm{rad} / \mathrm{s}$ : ac-side current (upper-left); $d q$ frame ac-side current (lower-left); PCC voltage (upper-center); circulating current (lower-center); upper- and lower-arm sum-capacitor-voltages (right).

the analyzed frequency range. An analysis of the individual terms of the current controller shows that the ac-side current closed-loop-system bandwidth reduces the admittance magnitude without degrading the phase, making it beneficial for the stability of the grid-converter system. On the other hand, the resonant term in the per-phase controller, the integral term in the $d q$ frame controller, and the PCC voltage feedforward term have mixed effects, as they lower the admittance magnitude but also increase the phase rotation around the fundamental frequency.

Computing the insertion indices using a closed-loop scheme drastically simplifies the ac-side admittance calculation, as the sum-capacitor voltages and the circulating current no longer appear as state variables. A closed-form expression is obtained, which coincides with the ac-side admittance of a two-level VSC. This compact expression can also be used as simplification for the open-loop scheme above fundamental frequency, assuming that current control is used. However, below fundamental frequency there is a noticeable difference between the two cases, therefore it is recommended to use the detailed model instead.

The findings from the admittance-shaping analysis are used to recreate a grid-converter system whose stability is determined by the value of the ac-side current closed-loop-system bandwidth. The developed admittance model is then used in an experimental case study, showing that the stability of the interconnected system can be assessed using the Nyquist stability criterion.

\section{APPENDIX}

\section{A. Steady-State Components of the MMC Variables}

The steady-state components of the converter variables used in the linear system (61) are given. Approximated solutions are used for simplicity, as they do not compromise the accuracy of the final result.

1) Upper-Arm and AC-Side Currents: Assuming that the current references (18) and (23) are tracked, it results

$$
I_{u}(0)=i_{c}^{\star} \quad I_{u}\left(f_{1}\right)=\frac{I_{s}\left(f_{1}\right)}{2} \quad I_{s}\left(f_{1}\right)=\frac{i_{s d}^{\star}+j i_{s q}^{\star}}{2} .
$$

2) Upper-Arm Insertion Index: Approximating the voltage references to the values assigned in (11), and neglecting the time delay, from (9) it results

$$
N_{u}(0)=\frac{1}{2} \quad N_{u}\left(f_{1}\right)=-\frac{e_{1}^{\star}}{2 v_{d}^{\star}} .
$$

3) Upper-Arm Sum-Capacitor-Voltage: The dc component of $v_{C u}^{\Sigma}$ is approximated to the dc-bus voltage reference and the fundamental-frequency component is obtained evaluating (5) at $f_{1}$, i.e.,

$$
V_{C u}^{\Sigma}(0)=v_{d}^{\star} \quad V_{C u}^{\Sigma}\left(f_{1}\right)=\frac{I_{u}\left(f_{1}\right) N_{u}(0)+N_{u}\left(f_{1}\right) I_{u}(0)}{j \omega_{1} C} .
$$

4) DQ Frame Voltage Reference: Expressing (7) in the $d q$ frame, assuming that $e_{1}=e_{1}^{\star}$ and that the current references (23) are tracked, gives

$$
V_{s d}^{\star}(0)=e_{1}^{\star}+\frac{R}{2} i_{s d}^{\star}-\frac{\omega_{1} L}{2} i_{s q}^{\star} \quad V_{s q}^{\star}(0)=\frac{R}{2} i_{s q}^{\star}+\frac{\omega_{1} L}{2} i_{s d}^{\star} .
$$

\section{B. Perturbation Components of the MMC Variables}

The linear expressions describing upper-arm current, upperarm voltage, and upper-arm sum-capacitor-voltage at $f_{p} \pm f_{1}$ and $f_{p} \pm 2 f_{1}$ are given. 

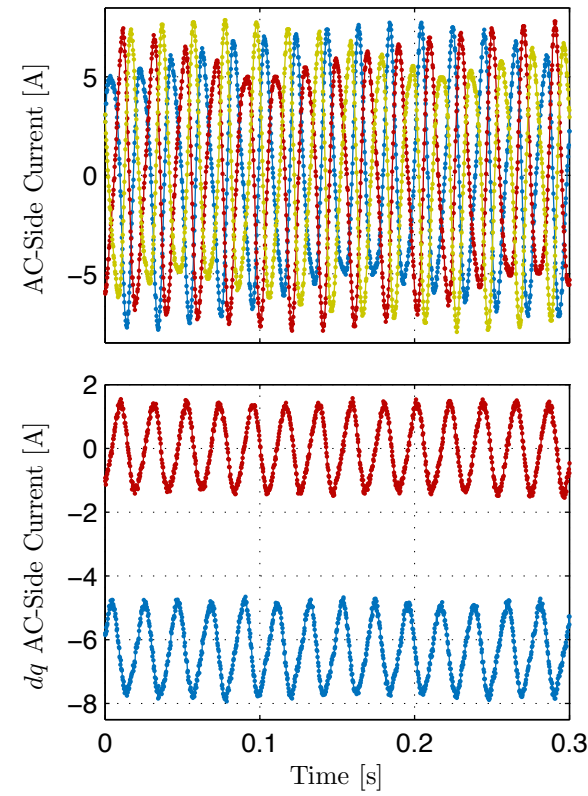
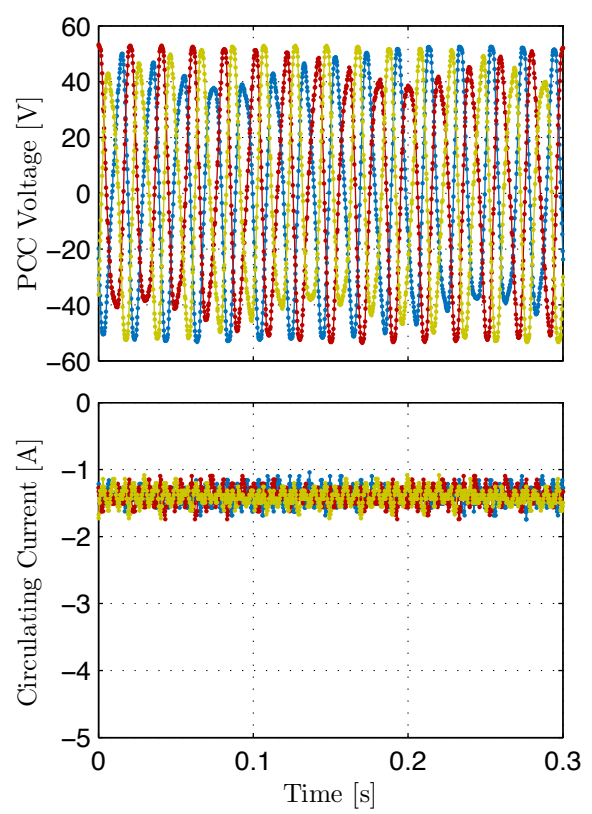
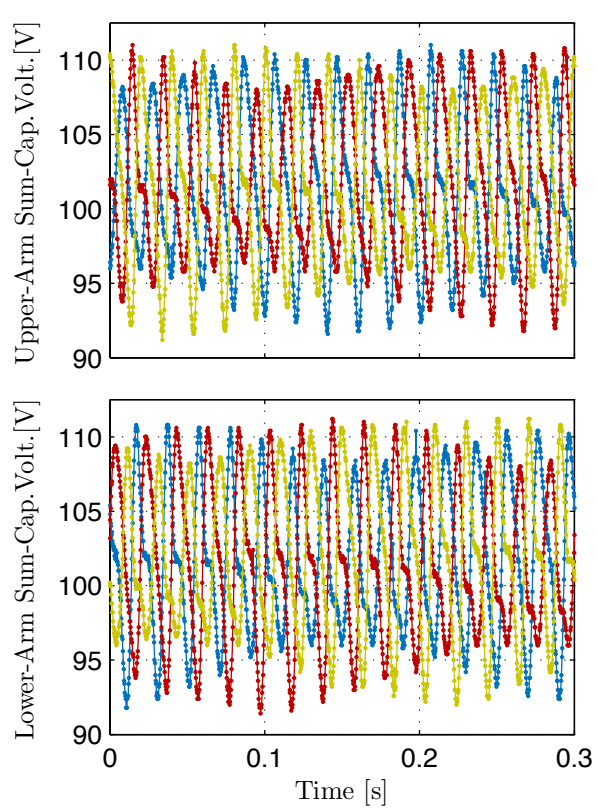

Fig. 18. Waveforms of the converter variables during the experiment with $\alpha_{s}=600 \mathrm{rad} / \mathrm{s}$ : ac-side current (upper-left); $d q$ frame ac-side current (lower-left); PCC voltage (upper-center); circulating current (lower-center); upper- and lower-arm sum-capacitor-voltages (right).

1) Upper-Arm Current: Obtained similarly to (30). Zerosequence components of $i_{u}$ flow into the de terminal; when using a resistive load on the dc side, the term $3 / 2 R_{d}$ must be included.

$$
\begin{gathered}
I_{u}\left(f_{p}-f_{1}\right)=-\frac{V_{u}\left(f_{p}-f_{1}\right)}{j\left(\omega_{p}-\omega_{1}\right) L+R+\frac{3}{2} R_{d}} \\
I_{u}\left(f_{p}+f_{1}\right)=-\frac{V_{u}\left(f_{p}+f_{1}\right)}{j\left(\omega_{p}+\omega_{1}\right) L+R} \\
I_{u}\left(f_{p}-2 f_{1}\right)=-\frac{V_{u}\left(f_{p}-2 f_{1}\right)}{j\left(\omega_{p}-2 \omega_{1}\right) L+R} \\
I_{u}\left(f_{p}+2 f_{1}\right)=-\frac{V_{u}\left(f_{p}+2 f_{1}\right)}{j\left(\omega_{p}+2 \omega_{1}\right) L+R+\frac{3}{2} R_{d}} .
\end{gathered}
$$

2) Upper-Arm Voltage: Obtained similarly to (31).

$$
\begin{aligned}
& V_{u}\left(f_{p}-f_{1}\right)=V_{C u}^{\Sigma}\left(f_{p}-f_{1}\right) N_{u}(0)+N_{u}\left(f_{p}-f_{1}\right) V_{C u}^{\Sigma}(0) \\
& \quad+V_{C u}^{\Sigma}\left(f_{p}-2 f_{1}\right) N_{u}\left(f_{1}\right)+N_{u}\left(f_{p}-2 f_{1}\right) V_{C u}^{\Sigma}\left(f_{1}\right) \\
& \quad+V_{C u}^{\Sigma}\left(f_{p}\right) \overline{N_{u}\left(f_{1}\right)}+N_{u}\left(f_{p}\right) \overline{V_{C u}^{\Sigma}\left(f_{1}\right)} \\
& \quad V_{u}\left(f_{p}+f_{1}\right)=V_{C u}^{\Sigma}\left(f_{p}+f_{1}\right) N_{u}(0)+N_{u}\left(f_{p}+f_{1}\right) V_{C u}^{\Sigma}(0) \\
& \quad+V_{C u}^{\Sigma}\left(f_{p}\right) N_{u}\left(f_{1}\right)+N_{u}\left(f_{p}\right) V_{C u}^{\Sigma}\left(f_{1}\right) \\
& \quad+V_{C u}^{\Sigma}\left(f_{p}+2 f_{1}\right) \overline{N_{u}\left(f_{1}\right)}+N_{u}\left(f_{p}+2 f_{1}\right) \overline{V_{C u}^{\Sigma}\left(f_{1}\right)}
\end{aligned}
$$

$$
\begin{aligned}
& V_{u}\left(f_{p}-2 f_{1}\right)=V_{C u}^{\Sigma}\left(f_{p}-2 f_{1}\right) N_{u}(0)+N_{u}\left(f_{p}-2 f_{1}\right) V_{C u}^{\Sigma}(0) \\
& \quad+V_{C u}^{\Sigma}\left(f_{p}-f_{1}\right) \overline{N_{u}\left(f_{1}\right)}+N_{u}\left(f_{p}-f_{1}\right) \overline{V_{C u}^{\Sigma}\left(f_{1}\right)} \\
& \quad \begin{array}{l}
V_{u}\left(f_{p}+2 f_{1}\right)=V_{C u}^{\Sigma}\left(f_{p}+2 f_{1}\right) N_{u}(0)+N_{u}\left(f_{p}+2 f_{1}\right) V_{C u}^{\Sigma}(0) \\
\quad+V_{C u}^{\Sigma}\left(f_{p}+f_{1}\right) N_{u}\left(f_{1}\right)+N_{u}\left(f_{p}+f_{1}\right) V_{C u}^{\Sigma}\left(f_{1}\right) .
\end{array}
\end{aligned}
$$

3) Upper-Arm Sum-Capacitor-Voltage: Obtained similarly to $(80)$.

$$
\begin{aligned}
& V_{C u}^{\Sigma}\left(f_{p}-f_{1}\right)=\frac{1}{j\left(\omega_{p}-\omega_{1}\right) C}\left[I_{u}\left(f_{p}-f_{1}\right) N_{u}(0)\right. \\
& \quad+N_{u}\left(f_{p}-f_{1}\right) I_{u}(0)+I_{u}\left(f_{p}-2 f_{1}\right) N_{u}\left(f_{1}\right) \\
& \left.\quad+N_{u}\left(f_{p}-2 f_{1}\right) I_{u}\left(f_{1}\right)+I_{u}\left(f_{p}\right) \overline{N_{u}\left(f_{1}\right)}+N_{u}\left(f_{p}\right) \overline{I_{u}\left(f_{1}\right)}\right]
\end{aligned}
$$

$$
\begin{aligned}
& V_{C u}^{\Sigma}\left(f_{p}+f_{1}\right)=\frac{1}{j\left(\omega_{p}+\omega_{1}\right) C}\left[I_{u}\left(f_{p}+f_{1}\right) N_{u}(0)\right. \\
& \quad+N_{u}\left(f_{p}+f_{1}\right) I_{u}(0)+I_{u}\left(f_{p}\right) N_{u}\left(f_{1}\right)+N_{u}\left(f_{p}\right) I_{u}\left(f_{1}\right) \\
& \left.\quad+I_{u}\left(f_{p}+2 f_{1}\right) \overline{N_{u}\left(f_{1}\right)}+N_{u}\left(f_{p}+2 f_{1}\right) \overline{I_{u}\left(f_{1}\right)}\right]
\end{aligned}
$$

$$
\begin{aligned}
& V_{C u}^{\Sigma}\left(f_{p}-2 f_{1}\right)=\frac{1}{j\left(\omega_{p}-2 \omega_{1}\right) C}\left[I_{u}\left(f_{p}-2 f_{1}\right) N_{u}(0)\right. \\
& \quad+N_{u}\left(f_{p}-2 f_{1}\right) I_{u}(0)+I_{u}\left(f_{p}-f_{1}\right) \overline{N_{u}\left(f_{1}\right)} \\
& \left.\quad+N_{u}\left(f_{p}-f_{1}\right) \overline{I_{u}\left(f_{1}\right)}\right]
\end{aligned}
$$

$$
\begin{aligned}
& V_{C u}^{\Sigma}\left(f_{p}+2 f_{1}\right)=\frac{1}{j\left(\omega_{p}+2 \omega_{1}\right) C}\left[I_{u}\left(f_{p}+2 f_{1}\right) N_{u}(0)\right. \\
& \quad+N_{u}\left(f_{p}+2 f_{1}\right) I_{u}(0)+I_{u}\left(f_{p}+f_{1}\right) N_{u}\left(f_{1}\right) \\
& \left.\quad+N_{u}\left(f_{p}+f_{1}\right) I_{u}\left(f_{1}\right)\right] .
\end{aligned}
$$

\section{REFERENCES}

[1] A. Lesnicar and R. Marquardt, "An innovative modular multilevel converter topology suitable for a wide power range," in Power Tech Conf. Proc., Bologna, Italy, 2003.

[2] S. Allebrod, R. Hamerski, and R. Marquardt, "New transformerless, scalable modular multilevel converters for HVDC-transmission," in 2008 IEEE Power Electron. Spec. Conf. IEEE, Jun. 2008, pp. 174-179.

[3] H. Akagi, "Classification, terminology, and application of the modular multilevel cascade converter (MMCC)," IEEE Trans. Power Electron. vol. 26, no. 11, pp. 3119-3130, Nov. 2011. 
[4] S. Debnath, J. Qin, B. Bahrani, M. Saeedifard, and P. Barbosa, "Operation, control, and applications of the modular multilevel converter: A review," IEEE Trans. Power Electron., vol. 30, no. 1, pp. 37-53, Jan. 2015.

[5] M. A. Perez, S. Bernet, J. Rodriguez, S. Kouro, and R. Lizana, "Circuit topologies, modeling, control schemes, and applications of modular multilevel converters," IEEE Trans. Power Electron., vol. 30, no. 1, pp. 4-17, Jan. 2015.

[6] M. Glinka and R. Marquardt, "A new ac/ac multilevel converter family," IEEE Trans. Ind. Electron., vol. 52, no. 3, pp. 662-669, Jun. 2005.

[7] M. Saeedifard and R. Iravani, "Dynamic performance of a modular multilevel back-to-back HVDC system," IEEE Trans. Power Deliv., vol. 25, no. 4, pp. 2903-2912, Oct. 2010.

[8] L. Harnefors, A. Antonopoulos, S. Norrga, L. Ängquist, and H.-P. Nee, "Dynamic analysis of modular multilevel converters," IEEE Trans. Ind. Electron., vol. 60, no. 7, pp. 2526-2537, Jul. 2013.

[9] M. Hagiwara and H. Akagi, "Control and experiment of pulsewidthmodulated modular multilevel converters," IEEE Trans. Power Electron., vol. 24, no. 7, pp. 1737-1746, Jul. 2009.

[10] M. Hagiwara, R. Maeda, and H. Akagi, "Control and analysis of the modular multilevel cascade converter based on double-star choppercells (MMCC-DSCC)," IEEE Trans. Power Electron., vol. 26, no. 6, pp. 1649-1658, Jun. 2011.

[11] S. Rohner, S. Bernet, M. Hiller, and R. Sommer, "Modulation, losses, and semiconductor requirements of modular multilevel converters," IEEE Trans. Ind. Electron., vol. 57, no. 8, pp. 2633-2642, Aug. 2010.

[12] Q. Tu, Z. Xu, and L. Xu, "Reduced switching-frequency modulation and circulating current suppression for modular multilevel converters," IEEE Trans. Power Deliv., vol. 26, no. 3, pp. 2009-2017, Jul. 2011.

[13] K. Ilves, A. Antonopoulos, S. Norrga, and H.-P. Nee, "Steady-state analysis of interaction between harmonic components of arm and line quantities of modular multilevel converters," IEEE Trans. Power Electron., vol. 27, no. 1, pp. 57-68, Jan. 2012.

[14] Q. Song, W. Liu, X. Li, H. Rao, S. Xu, and L. Li, "A steady-state analysis method for a modular multilevel converter," IEEE Trans. Power Electron., vol. 28, no. 8, pp. 3702-3713, Aug. 2013.

[15] M. Guan and Z. Xu, "Modeling and control of a modular multilevel converter-based HVDC system under unbalanced grid conditions," IEEE Trans. Power Electron., vol. 27, no. 12, pp. 4858-4867, Dec. 2012.

[16] A. Nami, J. Liang, F. Dijkhuizen, and G. D. Demetriades, "Modular multilevel converters for HVDC applications: Review on converter cells and functionalities," IEEE Trans. Power Electron., vol. 30, no. 1, pp. 18-36, Jan. 2015.

[17] J. Sun, "Impedance-based stability criterion for grid-connected inverters," IEEE Trans. Power Electron., vol. 26, no. 11, pp. 3075-3078, Nov. 2011.

[18] L. Harnefors, X. Wang, A. G. Yepes, and F. Blaabjerg, "Passivity-based stability assessment of grid-connected VSCs-An overview," IEEE $J$. Emerg. Sel. Top. Power Electron., vol. 4, no. 1, pp. 116-125, Mar. 2016.

[19] L. Harnefors, M. Bongiorno, and S. Lundberg, "Input-admittance calculation and shaping for controlled voltage-source converters," IEEE Trans. Ind. Electron., vol. 54, no. 6, pp. 3323-3334, Dec. 2007.

[20] X. Wang, L. Harnefors, and F. Blaabjerg, "Unified impedance model of grid-connected voltage-source converters," IEEE Trans. Power Electron., vol. 33, no. 2, pp. 1775-1787, Feb. 2018.

[21] M. Beza, M. Bongiorno, and G. Stamatiou, "Analytical derivation of the ac-side input admittance of a modular multilevel converter with openand closed-loop control strategies," IEEE Trans. Power Deliv., vol. 33, no. 1, pp. 1-1, Feb. 2017.

[22] J. Khazaei, M. B. Beza, and M. Bongiorno, "Impedance analysis of modular multi-level converters connected to weak ac grids," IEEE Trans. on Power Syst., vol. 33, no. 4, pp. 4015-4025, July 2018.

[23] J. Lyu, Q. Chen, and X. Cai, "Impedance modeling of modular multilevel converters by harmonic linearization," in 2016 IEEE 17th Work. Control Model. Power Electron., Jun. 2016.

[24] J. Lyu, X. Cai, and M. Molinas, "Optimal design of controller parameters for improving the stability of MMC-HVDC for wind farm integration," IEEE J. Emerg. Sel. Top. Power Electron., pp. 1-1, 2017.

[25] J. Lyu, X. Zhang, X. Cai, and M. Molinas, "Harmonic state-space based small-signal impedance modeling of modular multilevel converter with consideration of internal harmonic dynamics," IEEE Trans. Power Electron., pp. 1-1, 2018.

[26] J. Sun and H. Liu, "Sequence impedance modeling of modular multilevel converters," IEEE J. Emerg. Sel. Top. Power Electron., vol. 5, no. 4, pp. 1427-1443, Dec. 2017.
[27] L. Bessegato, L. Harnefors, K. Ilves, and S. Norrga, "A method for the calculation of the ac-side admittance of a modular multilevel converter," IEEE Trans. Power Electron., pp. 1-1, 2018.

[28] K. Sharifabadi, L. Harnefors, H.-P. Nee, S. Norrga, and R. Teodorescu, Design, control and application of modular multilevel converters for HVDC transmission systems. Chichester, UK: John Wiley \& Sons, Ltd, 2016.

[29] Z. Li, P. Wang, Z. Chu, H. Zhu, Y. Luo, and Y. Li, "An inner current suppressing method for modular multilevel converters," IEEE Trans. Power Electron., vol. 28, no. 11, pp. 4873-4879, Nov. 2013.

[30] A. Antonopoulos, L. Angquist, and H. Nee, "On dynamics and voltage control of the modular multilevel converter," in 2009 13th Eur. Conf. Power Electron. Appl. EPE'09 ECCE Eur., Sep. 2009.

[31] J. Sun, "Small-signal methods for ac distributed power systems-A review," IEEE Trans. Power Electron., vol. 24, no. 11, pp. 2545-2554, Nov. 2009.

[32] L. Bessegato, A. Narula, P. Bakas, and S. Norrga, "Design of a modular multilevel converter prototype for research purposes," in 2018 20th Eur. Conf. Power Electron. Appl. EPE'18 ECCE Eur., Sep. 2018.

[33] K. Ilves, L. Harnefors, S. Norrga, and H.-P. Nee, "Analysis and operation of modular multilevel converters with phase-shifted carrier PWM," IEEE Trans. Power Electron., vol. 30, no. 1, pp. 268-283, Jan. 2015. 